\title{
Revisión y propuestas de intervención sobre el Efectode la Edad Relativa en losámbitos educativo y deportivo \\ Review of relative age effects and potential ways to reduce them in sport and education
}

\author{
David Gutiérrez Díaz del Campo \\ Universitad de Castilla-La Mancha
}

\begin{abstract}
Resumen: La diferencia de edad cronológica entre los sujetos de un mismo grupo de edad es conocido como edad relativa (relative age) y sus consecuencias como efecto de la edad relativa (RAE: Relative age effect). Esta expresión ha sido en diversos trabajos mal traducida al castellano como efecto relativo de la edad. En el presente trabajo se exponen las investigaciones más relevantes realizadas en torno a este tema tanto en el ámbito deportivo como en el educativo a nivel mundial, con especial atención a los estudios en español. Se ha elegido realizar una revisión conjunta de los ámbitos educativo y deportivo por existir nuemerosos puntos en común, así como de tratarse en ocasiones de los mismos sujetos los incluidos en unos y otros estudios, pues todos los deportistas han formado parte del ámbito educativo en su etapa obligatoria. Este trabajo no pretende ser una revisión exahustiva del RAE, sino de aquellos trabajos más representativos en cada uno de los ámbitos revisados y los subámbitos que aparecen en cada uno de llos. En la parte final del artículo se exponenen y analizan las hipótesis barajadas como origen de este problema, así como las soluciones propuestas en la bibliografía, además de propuestas propias elaboradas por el autor. Más allá de aportar soluciones genéricas, se pretende generar sensibilidad hacia este problema y voluntad de intervención en educadores y responsables deportivos.
\end{abstract}

Palabra clave: efecto de la edad relativa; efecto relativo de la edad; detección de talentos; procesos de selección.

Abstract: Differences on chronological age within a peer/age group are known as Relative Age, and its consecuences as Relative Age Effec (RAE). This article is a review of the most relevant publications in both the educative and sportive contexts. The decision of reviewing both contexts in the same article is based on the existence of many points in common between them. Furthermore when research is made on youth sport, all the participants are at the same time in the educational system. The hypothesis of the origin of RAE and possible solutions are discussed and analysed at the end of the paper. Besides the objective of compiling and analyse what literature says about RAE, this article has the aim of raisig awareness about this problem and wish of intervention in educators and sportive managers.

Key words: relative age effect; season-of-birth bias; selection processes; talent identification.

\section{Objetivos del Artículo}

Realizar una revisión de las investigaciones realizadas sobre el efecto de la edad relativa en el ámbito educativo y en el deportivo y establecer conexiones entre ambos ámbitos de investigación.

Exponer las principales causas subyacentes a las desigualdades producidas por el efecto de la edad relativa.

-Exponer la importancia que este factor supone en la formación y promoción de jugadores dentro de una escuela, o club deportivo.

Exponer distintas propuestas de actuación para evitar o al menos mitigar los efectos negativos de la edad relativa.

- Más allá de aportar soluciones genéricas, se pretende generar sensibilidad hacia este problema y voluntad de intervención en educadores y responsables deportivos.

\section{Introducción: Definición y Corrección del Término Español}

Existen diversos ámbitos en los que se requiere el agrupamiento por edad de los sujetos, como el sistema educativo o el deportivo, la finalidad de este tipo de organización tiene como objetivo la justa igualdad de oportunidades cuando se trata del ámbito competitivo y asegurar un proceso de formación estándar lo más adecuado posible a todos los sujetos en el caso del ámbito educativo. No obstante, siempre existirán diferencias de edad y por lo tanto potencialmente de maduración y experiencia entre los integrantes de la categoría, curso o grupoen cuestión. La diferencia de edad cronológica entre los sujetos de un mismo grupo de edad es conocido como edad relativa (relative age) y sus consecuencias como efecto de la edad relativa (a patir de ahora RAE: Relative Age Effect).

Las consecuencias del RAE han sido evidenciadas en diversos ámbitos, pudiéndose interpretar como una discriminación sistemática o desigualdad de oportunidades de los individuos nacidos poco antes de la

Fecha recepción: 30-04-12 - Fecha envío revisores: 30-04-12 - Fecha de aceptación: 25-05-12 Correspondencia: David Gutiérrez Díaz del Campo

Ronda de Calatrava 3

Ronda de Calatrava 3

E-mail: david.gutierrez@uclm.es fecha de corte del año competitivo de selección o escolar. Esta discriminación tiene como potenciales consecuencias, para aquellos que su edad relativa es menor que la de sus compañeros, peores resultados académicos, mayor probabilidad de ser evaluado como alumno con necesidades educativas especiales, mayor provabilidad de adquirir incompetencia aprendida, menor participación en actividades deportivas, mayor porcentaje de abandono de la práctica deportiva y menor probabilidad de ser elegido en los procesos de detección, así como de acceder a los primeros equipos mediante los filtrados de selección que se dan dentro de los clubes.

En elpresente trabajo se expondrán las investigaciones más relevantes realizadas en torno a este tema, superando esta revisión al ámbito deportivo, y abordando también el educativo por existir nuemerosos puntos en común, así como de tratarse de los mismos sujetos los incluidos en unos y otros estudios (todos los deportistas han formado parte del ámbito educativo en su etapa obligatoria). En los siguientes apartados se realizará una revisión de las hipótesis barajadas como origen de este problema, así como de las soluciones propuestas en la bibliografía, además de propuestas propias elaboradas por el autor.

\subsection{Aclaración Terminológica}

En diversos textos publicados en español (e.g. García Álvarez \& Salvadores Canedo, 2005; González Aramendi, 2007; Martín Acero, et al., 2007) se recoge la expresión de efecto relativo de la edad, como traducción de la expresión inglesa relative age effect. Esta traducción es incorrecta. El efecto descrito es el producido por la edad relativa (edad relativa: diferencia de edad cronológica entre los sujetos de un mismo grupo de edad), y no es el efecto el que es relativo (efecto de la edad relativa es un sintagma dentro del cual el adjetivo relativo modifica al sustantivo edad no a efecto). De hecho, si tradujesemos efecto relativo de la edad al inglés, la expresión resultante sería: relative effect of age. Existen no obtante publicaciones, especialmente las más recientes, en las que se ha corregido este error (e.g. Esteva, Drobnic, Puigdellivo, Serratosa \& Chamorro, 2006; Gómez Piqueras, 2009; Lesma, Pérez González \& Salinero, 2011), y en las que ya aparece la expresión efecto de la edad relativa. Debido a esta controversia y a que lo realmente generalizado es la expresión inglesa, especialmente el acrónimo (RAE), utilizaremos dicho acrónimo en la presente revisión. 


\section{Revisión Bibliográfica: Antecedentes y Estado Actual de la Investigación}

Los ámbitos en los que se da el RAE son aquellos en los que existen agrupamientos por edad y por lo tanto también fechas de corte. De forma genérica podemos decir que los tres grandes terrenos en los que esto se da son en el académico, el deportivo y el clínico, concretamente en la psicología clínica. En esta revisión nos centraremos en los dos primeros. En la enseñanza reglada de la mayoría de los países se establecen agrupaciones anuales, siendo la fecha de inicio del año de selección mayoritariamente la del 1 de enero, aunque también está extendida, sobre todo en los países anglosajones, la del inicio del curso escolar, es decir, el 1 de septiembre. En el caso de Estados Unidos esta fecha varía de un estado a otro; en Bedard \& Dhuey (2005) podemos encontrar un listado de la fecha de inicio del año de escolaridad de numeros países así como de los disitntos estados de Estados Unidos. En el ámbito deportivo se da la misma situación, salvo que el 1 de enero está globalmente más aceptado como inicio del año de selección, aunque en ciertos deportes de países como Bélgica, Australia o Estados Unidos se han utilizado o utilizan otras, como el 1 de agosto o 1 de septiembre.

\section{1. Ámbito Educativo}

Dentro del ámbito académico encontramos diversos campos de investigación en los que el RAE ha sido la variable central a estudiar. Se exponen a continuación las principales investigaciones agrupadas en torno a los siguientes temas: logros académicos, problemas de aprendizaje, superdotación, liderazgo, educación física, deporte escolar, autoestima y suicidios.

\subsubsection{Logros académicos.}

En este ámbito existen numerosos estudios sobre el RAE, no habiéndose encontradoen todos ellos evidencias significativas. La mayoría de las investigaciones que se han centrado en los primeros cursos han hallado diferencias significativas entre los logros alcanzados por los alumnos nacidos cerca de la fecha de corte y los que son casi un añomás pequeños que estos (e.g. Cameron \& Wilson, 1990; Crosser, 1991; Davis, Trimble \& Vincent, 1980), existiendo diferencias tanto en los logros académicos en general (e.g. Breznit \& Teltsch, 1989; Cameron \& Wilson, 1990; Crosser, 1991; Davis, Trimble \& Vincent, 1980; Lien, Tambs, Oppedal, Heyerdahl \& Bjertness, 2005;Thompson, 1971) como en capacidades específicas tales como la lectura (Strøm, 2003). También se han hallado diferencias significativas en el porcentaje de alumnos que terminan sus estudios (Peck \& Trimmer, 1995). Sin embargo ha habido estudios realizados en diferentes edades que no han encontrado diferencias significativas en cuanto a los logros escolares (Dietz \& Wilson, 1985; Kinard, Reinhertz, 1986; Peck \& Trimmer, 1995). A continuación se resumen dos estudios representativos sobre este tema, los estudios realizados por Sprietsma (2006) y por Bedard y Dhuey (2006). En ambos se puede deducir una posible transferencia al deporte.

Sprietsma (2006) realiza una investigación sobre los resultados académicos mostrados por alumnos de 16 países en las materias de lectura y matemáticas. La importancia de este artículo es el intento de constatación del RAE a largo plazo en un ámbito en el que no existen abandonos, puesto que se trata de evaluar a alumnos al final de la educación obligatoria, por lo tanto la composición de la muestra no variaría de una edad a otra. Esta circunstancia es importante porque es uno de los problemas a los que nos enfrentamos cuando realizamos un estudio en el ámbito deportivo, el abandono, ya que elabandono deportivo interfiere en la interpretación de datos en estudios longitudinales del RAE puesto que modifica la muestra. Sin embargo, en este estudio tenemos la posibilidad de contar con todos los sujetos cuando ya han superado una gran parte de su formación académica, pudiendo comprobar si los alumnos más jóvenes tienen mayores dificultades a lo largo de todo el proceso.

A continuación se exponen los canales a través de los que se podría manifestar el RAE según Sprietsma (2006):
-Mayor probabilidad de repetir curso. Se da en 6 de los 11 países estudiados, donde ser de los mayores de la clase reduce la probabilidad de repetir entre un 2 y un $15 \%$. Otros factores que aumentan la probabilidad de repetir curso: ser chico, padres divorciados, número de libros en casa.

- Escoger el itinerario deseado: el RAE sólo afecta en 2 de los 7 países estudiados. El factor que más influye es la estructura familiar y el nivel de formación de los padres.

- Alumnos que están en curso superior al que le corresponde (suben de categoría). La mayor parte de estos son lo nacidos en el primer trimestre.

Tabla 1. Resumen del efecto de la normativa macional de admisión para la enseñanza primaria (RELAGE) y los canales a través de los cuales se manifiesta, Sprietsma (2006).

\begin{tabular}{|c|c|c|c|c|c|c|}
\hline \multirow[b]{2}{*}{ Country } & \multirow{2}{*}{$\begin{array}{l}\text { Total effect } \\
\text { of RELAGE }\end{array}$} & \multicolumn{4}{|c|}{ Channels through which it occurs } & \multirow[t]{2}{*}{ Remarks } \\
\hline & & Voced Earlystart & Latestart & Retention & Grade too high & \\
\hline Belgium & Positive & $\mathrm{x}$ & $\mathrm{x}$ & $\mathrm{x}$ & $\mathrm{x}$ & \\
\hline Canada & Positive & & & $\mathrm{x}$ & $\mathrm{x}$ & \\
\hline Denmark & Positive & & $\mathrm{x}$ & & $\mathrm{x}$ & \\
\hline France & None & $\mathrm{x}$ & & & $\mathrm{x}$ & No school info \\
\hline Iceland & None & & & & & \\
\hline Italy & Positive & $\mathrm{x}$ & $\mathrm{x}$ & $\mathrm{x}$ & $\mathrm{x}$ & \\
\hline Japan & Positive & & & & & \\
\hline Korea & Positive & & & & & Only for readin \\
\hline Latvia & Positive & $\mathrm{x}$ & & & $\mathrm{x}$ & \\
\hline New Zealand & Positive & & $\mathrm{x}$ & & $\mathrm{x}$ & \\
\hline Norway & Positive & $\mathrm{x}$ & & & & Only for readin̨̧ \\
\hline Poland & None & $\mathrm{x}$ & & & & \\
\hline Portugal & None & & $\mathrm{x}$ & $\mathrm{x}$ & $\mathrm{x}$ & \\
\hline Spain & Positive & & $\mathrm{x}$ & $\mathrm{x}$ & & \\
\hline Sweden & Positive & & $\mathrm{x}$ & & $\mathrm{x}$ & \\
\hline Yugoslaria & None & & & & $\mathrm{x}$ & \\
\hline
\end{tabular}

Las principales conclusiones derivadas de este estudio son:

- La existencia de RAE a lo largo de todo el proceso educativo en la mayoría de los países analizados.

- La no confirmación absoluta del RAE debido a la agrupación por habilidad.

-Formulación de la hipótesis sobre el papel positivo de los profesores en países donde no existe RAE.

- Repetición de curso: es una variable que lleva a obtener peores resultados en los test y los alumnos más pequeños son más propensos a repetir.

De los 16 países estudiados, 10 mostraron clara presencia del RAE durante todo el proceso educativo. Según los autores esto es debido a la agrupación de los sujetos mediante un criterio de rendimiento, como es el de repetir curso, pero existen países en los que se da la agrupación por habilidad yno se ha encontradoevidencias de RAE. Los autores justifican estos datos por el posible papel jugado por los profesores en cuanto a sus expectativas con respecto a los resultados de sus alumnos. Afirman que esta variable está descrita en estudios científicos en la literatura sobre psicología y que datos que validasen esta hipótesis en posteriores investigaciones podrían justificar un cambio en la política educativa para que se invirtiera más en la concienciación y formación de los profesores en este sentido.

En el análisis de este trabajo encontramos varias posibles transferencias en el estudio del RAE al ámbito deportivo:

Estudio sobre la influencia del RAE en la elección del deporte.

Estudio del RAE en jugadores que son ascendidos de categoría o que entran en convocatorias de los primeros equipos.

-Estudiode casos en torno a lainfluencia de la laborde los entrenadores y formadores en la mitigación o en el incremento del RAE.

Otro estudio de gran interés es el realizado por Bedard y Dhuey (2006). Estos autores realizan un gran trabajo en el seguimiento longitudinal del impacto de la edad relativa a lo largo del proceso educativo en diferentes paises, de tal forma que analizan los resultados académicos de los estudiantes desde los primeros años de escolarización hasta el final de la educación obligatoria, haciendo un seguimiento hasta los estudios superiores en los casos de Estados Unidos e Inglaterra. Para Berdad y Dhuey el hechode que algunos alumnos sean aproximadamente un veinte por ciento mayores cronológicamente que los más jóvenes de su mismo grupo al inicio de la escolarización es el responsable de un 
efecto duradero y a largo plazo en los logros escolares, dependiendo las diferencias encontradas entre los más jóvenes y los mayores del país en el que se mida. En Inglaterra y en NuevaZelanda los resultados muestran que los sujetos tienen incluso menos posibilidades de completar sus estudios. La demostración de RAE a más largo plazo se concreta en este estudio en que los alumnos mayores de cada grupo tienen mayor probabilidad de realizar programas preuniversitarios durante el último año de educación secundaria, y con mayores posibilidades de ingresar en una institución universitaria de prestigio en Estados Unidos. Un aspecto interesante de este estudio es que muestra una de las grandes dificultades a las que se enfrentan los investigadores a la hora de analizar el RAE en un ámbito determinado: los sujetos que aún nacidos al final del año de selección son los mayores del grupo. Esto puede ser debido a la repetición de curso o al retraso voluntario, por parte de los padres en la escolarización. Lo que se pretende con ello es precisamente invertir el efecto de la edad relativa, buscando que el niño se vea favorecido por éste. Si bien en nuestro país esto no ocurre en el ámbito deportivo, sí que se da en otros países, donde las agrupaciones deportivas se realizan en torno al año académico y no de nacimiento. Este fenómeno ha sido denominado como «redshirting» (camiseta roja). Este término fue adoptado por el ámbito académico después de ser utilizado en el deportivo, ya que a estos sujetos se les identificaba en las competiciones con una camiseta roja. Existen estudios sobre fútbol americano que relacionan este fenómeno con la intención de los padres de favorecer a sus hijos en este deporte.

Lo más interesante de este estudioen cuanto a su posible transferencia al ámbito deportivo es la constatación de la perpetuación de los efectos negativos de la edad relativa a lo largo del proceso de formación En cuanto a la prolongación del efecto a lo largo del tiempo y no de su atenuación y desaparición como cabría suponerse. Estos autores afirman que debería prestarse más atención a las estructuras escolares que afectan a la adquisición de habilidades, estructuras tales como los tipos de agrupación, fecha de entrada, criterios de promoción, etc. Como única solución viable proponen una fecha de entrada semestral, el resto de posibilidades de solución que proponen las analizan y desechan. Añaden que sería muy interesante analizar las diferencias entre los efectos a largo plazo en aquellos países en los que no se realizan agrupaciones por rendimiento, como Finlandia o Dinamarca, frente a los que sí, como Estados Unidos e Inglaterra.

Como se puede comprobar, ambos estudios están preocupados por los efectos que producen los agrupamientos por rendimiento observado, si bien no son capaces de concluir nada definitivo, sino la probable incidencia de éstos en el aumento del RAE. Este es un asunto que también hasido debatido en el ámbitodeportivo, existiendo diferentes posturas; mientras algunos autores consideran que este tipo de agrupaciones es uno de los factores potenciadotes del RAE, puesto que los jugadores que nos son seleccionados para los primeros equipos tiene mayor probabilidad de abandonar o de no alcanzar todo su potencial, Kaiserman (2005) expone su convencimiento de que esta es la solución al RAE, además de poder obtenerse otros beneficios de una agrupación por habilidad, si bien lo recomienda únicamente para ligas recreativas.

3.1.2. Problemas de aprendizaje o académicos específicos (specific learning disabilities: SLD).

Numerosos estudios se han realizado para determinar si la edad relativa afecta a la posibilidad de ser diagnosticado con necesidades educativas especiales, o de problemas de aprendizaje en general, siendo en la mayoría de los casos positivo el resultado (e.g. Bell \& Daniel, 1990; DeMeis \& Stears, 1992; Diamond, 1983; Gilly, 1965; Grondin, Proulx \& Zhou, 1993; Maddux, 1980; Hauck \& Finch, 1993; Livingston, Balkozar \& Bracha, 1993). El RAE ha sido investigado en estudios relacionados con la superdotación (Maddux, Stacy \& Scout, 1981) en los que los alumnos calificados como superdotados estaban presentes en mayor porcentaje entre los nacidos en el primer semestre del año.

\subsubsection{Liderazgo.}

Un aspecto relacionado con la superdotación es el del liderazgo, de esta forma la probabilidad de convertirse en líder de grupo ha sido estudiada en varios trabajos (para más información verla revisión realizada por Dixon, Horton \& Weir, 2011). Dhuey y Lipscomb (2006) determinaron que la actividad de liderazgo en la educación secundaria estaba asociada puramente a la estructura escolar, y no a características genéticas o características familiares. Las estructuras escolares al determinar una fecha de corte, serían las causantes de la introducción sistemática de variaciones en los niveles de maduración de los individuos, lo cual generaría diferencias en la capacidad de liderazgo. Los determinantes seleccionados para establecer la capacidad de liderazgo de los estudiantes fueron mediante la consecución de roles como el de presidente de club o capitán de equipo. Dhue y Lipscomb encontraron que los estudiantes nacidos en el primer trimestre del año escolar tienen cinco veces más probabilidades de convertirse en líderes que sus compañeros más jóvenes nacidos en el último trimestre.

\subsubsection{Educación Física y depote escolar.}

Siguiendo en elámbitoescolar encontramosinvestigaciones realizadas acerca de la actividad físico deportiva, incluyendo en este apartado los logros en Educación Física y en el deporte escolar. Sobre la Educación Física, Bell, Massey y Dexter (1997) estudiaron el efecto del RAE en los resultados optenidos en el GCSE (General Certificate of Secondary Education. Certificado de secundaria para cada asignatura en el Reino Unido, alumnos de 16 años. La calificación va desde la $\mathrm{G}$ a la A.)

Los resultaron mostraron que había RAE tanto en la parte física de la prueba como en la escrita, tanto en chicos como en chicas. En el deporte escolar las investigaciones se han centrado en la participación de los alumnos en las competiciones escolares (Glamser \& Marciani, 1992; Wilson, 1999) encontrandose evidencias de RAE pero con dependencia del deporte analizado, además de otros factores tales como la región o la raza (Glamser \& Marciani, 1992). Un ejemplo de estos estudios es el realizado por Wilson (1999) en el que se estudió la composición de los equipos de escuelas de enseñanza secundaria inglesas (comprehensive school), en los deportes de rugby, hockey, fútbol y deportes de red. Los nacidos en otoño, los mayores dentro de su grupo, fueron los que componían en un mayor porcentaje estos equipos escolares, existiendo la mayor diferencia con los nacidos en verano. Uniendo ambos ámbitos, los logros en Educación Física y la participación en el deporte escolar, Cobley (2005) y Cobley, Abraham y Baker (2008) realizan una investigación en la que analizan a 663 alumnos de educación secundaria en el ámbito físico deportivo, englobando las puntuaciones obtenidas en Educación Física, la participación en equipos deportivos y en la identificación de alumnos como talentos dentro del ámbito físico deportivo. En cada uno de estos apartados se encontró evidencia de RAE. Roberts y Stuart (2012) en un reciente estudio sobre la influencia delRAE y las calificaciones en Educación Física encontraron un alto nivel de significatividad en alumnos de 11 a 14 años.

\subsubsection{Autoestima en escolares.}

Se obtuvo evidencia de RAE en estudios como el realizado por Spitzer, Cupp y Parke (1995) sobre la relación que tiene la edad relativa en la aceptación social y la autopercepción en los primeros niveles educativos, o como los realizados por Bickel, Zigmond y Strayhorn (1991) o por Pellegrini (1992) ambos asociando la autoestima a la edad y su repercusión en los logros académicos. La siguiente gráfica muestra la relación entre la autoestima, medida mediante el Culture Free SelfEsteem Inventory (Battle, 1981 en Thompson et al., 2004) y la edad de entrada en la escuela. Se observa que el índice de autoestima muestra un general aumento en aquellos que están dentro del agrupamiento correcto según la edad. Los más altos niveles de autoestima fueron alcanzados por el grupo de alumnos que en su mayor parte estaban compuestos por alumnos que estaban destinados a ser los más pequeños de su clase, pero que, aparentemente por lo decisión de sus padres, retrasaron el ingreso en el colegio un año, haciéndolos de este modo los mayores de su curso. En la gráfica se muestra que la edad de entrada en la escuela marca el patrón del índice de autoestima, pero es la estructuración o no del hogar familiar lo que determina en mayor grado este aspecto de la personalidad. 


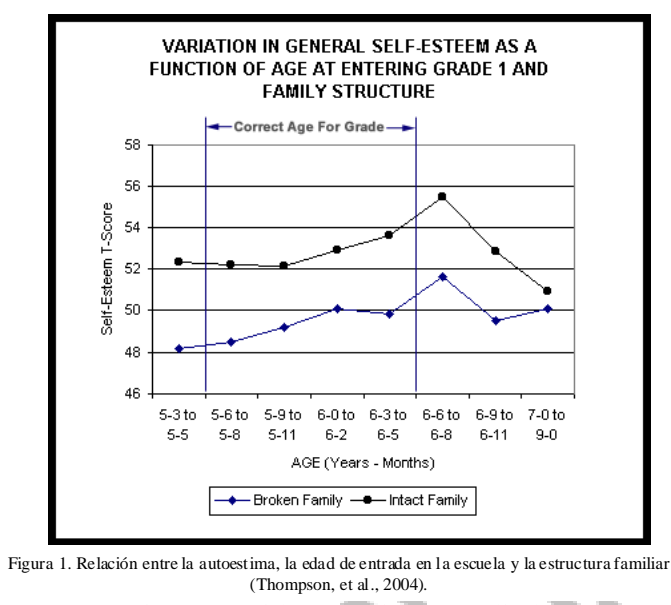

La autoestima ha sido también estudiada dentro de las investigaciones sobreelefecto de laedad relativa en cuantoa su importancia en el índice de suicidios (Thompson et al., 2004; Thompson, Barnsley \& Dyck, 1999). Thomson et al. (1999) demostraron la relación entre el suicidio de adolescentes y la edad relativa en la que estos individuos ingresaban en la educación formal. Se demostró que el ser más joven que los compañeros de grupo estaba relacionado con una disminución de la autoestima en años posteriores. Esto sugiere que la autoestima, o el factor relacionado de la percepción de la propia eficacia, sirve como nexo en la relación entre el RAE y el suicidio. Si bien el índice de suicidios o la autoestima no son temas académicos, los hemos incluido por estar relacionados con los logros académicos y porque estos estudios han sido realizados siempre en ámbitos y población eslcolar.

Tabla 2. Suicidios de jóvenes desde 1979 a 1992 en Alberta (Canadá) clasificados según la edad relativa y la fecha de inicio del año escolar (Thomson, et al., 1999).

\begin{tabular}{ccc}
\multicolumn{2}{c}{ del año escolar (Thomson, et al., 1999). } \\
\hline $\begin{array}{c}\text { Fecha de inicio } \\
\text { año escolar }\end{array}$ & $\begin{array}{c}\text { Nacidos en el } \\
\text { segundo semestre }\end{array}$ & $\begin{array}{c}\text { Nacidos en el } \\
\text { primer semestre }\end{array}$ \\
Mar 1 & 172 & 152 \\
Ene 1 & 117 & 92 \\
Sept 1 & 9 & 5 \\
Nov 1 & 7 & 2 \\
Oct 1 & 6 & 1 \\
Dic 1 & 1 & 0 \\
Total & 312 & 252 \\
\hline
\end{tabular}

\section{2. Ámbito Deportivo}

El RAE ha sido estudiado en un gran número de deportes, sin embargo por encima del resto destacan dos, el fútbol y el hockey sobre hielo, siendo este último en el que tiene sus orígenes este campo de investigación en el deporte.

\subsubsection{Hockey hielo.}

En hockey hielo el RAE ha sido investigado principalmente en Estados Unidos y Canadá. Uno de los primeros estudios fue el de Barnsley, Thompson y Barnsley (1985). La apertura de este campo de investigación fue motivada por investigaciones similares en el campo de la educación (Hurley, Lior \& Tracze, 2001). El RAE ha sido estudiado y evidenciado tanto en las ligas profesionales (Barnsley, Thompson \& Barnsley, 1985; Daniel \& Janssen, 1987; Grondin, Deshaies \& Nault, 1984, Grondin \& Trudeau, 1991) como en las competiciones inferiores (minor y major junior) (Barnsley \& Thomson, 1988; Boucher \& Halliwell, 1991; Boucher \& Mutimer, 1994; Grondin etal., 1984; Hurley, et al., 2001; Krouse, 1995). Aunque la mayoría de estas investigaciones aportan evidencias contundentes sobre la generalización del RAE, existen factores que deben ser tenidos en cuenta, como la edad, la categoría, la temporada o incluso la posición dentro del campo de juego. En la siguiente gráfica, extraida de Barnsley et al. (1985), se muestra la distribución de la fecha de nacimientode jugadores en dos ligas canadienses: Ontario Junior Hockey League y la Wester Hockey League. Los datos muestran que la probabilidad de alcanzar estas ligas superiores se reduce de una forma drástica para aquellos nacidos al final del año.

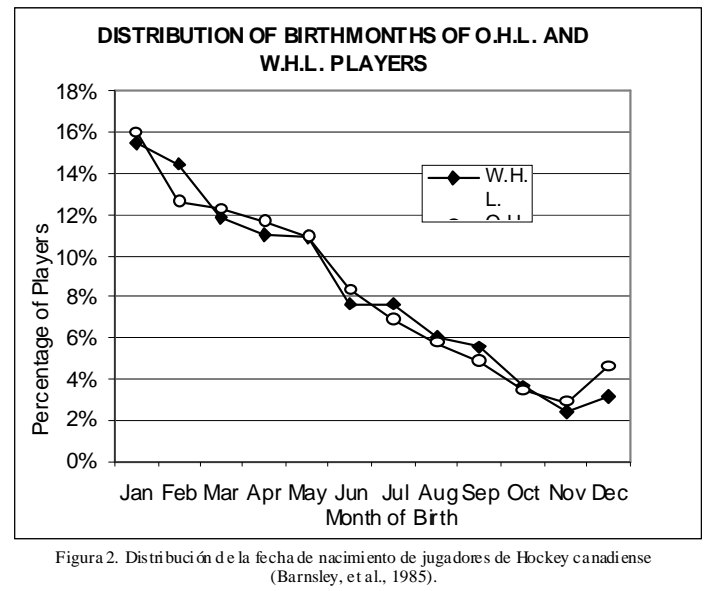

En la siguiente gráfica se muestra la distribución por fechas de nacimiento de una muestra de 8.000 jugadores de entre 9 y 15 años, en las tres divisiones en las que se organiza la Canadian Minor Hockey League, observándose una gran derivación de los nacidos al principio del calendario competitivo hacia las divisiones superiores. Los más jóvenes muestran una tendencia contraria.

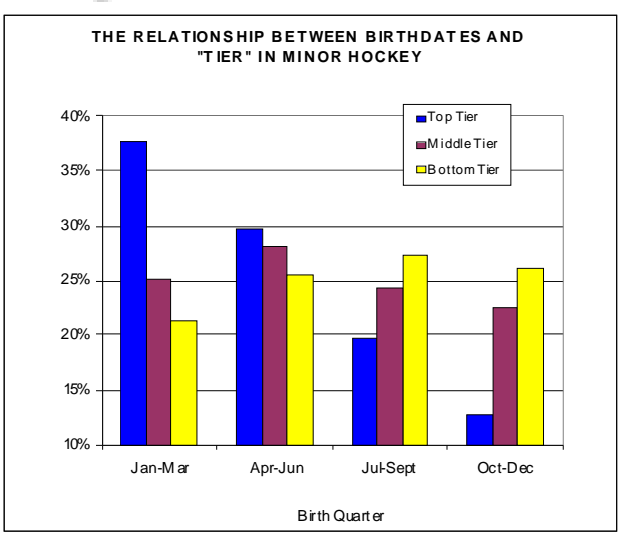

Figura 3. Rel ación entre fecha de nacimiento y categoría en M inor Hockey (Barnsley \& Thompson, 1988).

\subsubsection{Fútbol.}

El otro deporte extensamente estudiado ha sido el fútbol. En este caso, la casi mundial popularidad del fútbol ha hecho que el RAE haya sido investigado de forma prácticamente global. Ejemplos de estudios sobre las ligas profesionales de diferentes países son los realizados en Bélgica (Helsen, Winckel \& Willians, 2005; Helsen, Janet, Starkes \& Winckel, 1998; Helsen, Janet, Starkes \& Winckel, 2000), en Inglaterra (Dudink, 1994), Alemania (Augste \& Lames, 2011; Bäumler, 2000) o España (e.g. García Álvarez \& Salvadores Canedo, 2005; Gutierrez Díaz del Campo, Pastor Vicedo, González Víllora \& Contreras Jordán, 2010; Martín Acero, et al., 2007; Pérez Jiménez \& Matthew, 2008). Así como aquellos que correlacionan los datos de diferentes países, ya sea en sus competiciones correspondientes o en competiciones internacionales analizando las selecciones nacionales. Realizaremos primero una revisión de los estudios más importantes excluyendo los realizados con futbolistas españoles, a los que dedicaremos un apartado exclusivo más adelante.

Entre los estudios con clubes profesionales de distintos países está el de Verhulst (1992) quien estudió el RAE en Bélgica, con un 55\% de nacidos en el primer semestre, Francia, con un $58 \%$ y Holanda, con un $60 \%$. Otro ejemplo es la investigación realizada por Musch y Hay (1999) quienes analizaron la fecha de nacimiento de profesionales de Australia, Brasil, Alemania y Japón, siendo este último país en el que mayor descompensación encontraron, con un $66 \%$ de nacidos en el primer semestre y Alemania en el que menos, un 56\%. La otra vertiente de los estudios internacionales son aquellos realizados en competiciones 
internacionales, estudios realizados en mundiales como el de Barnsley et al. (1992) quienes constataron en el mundial de 1990 un 55\% de nacidos en el primer semestre entre los participantes. En la misma publicación se exponen los resultados hallados en los mundiales sub 17 y sub 20, siendo los resultados mucho más extremos en cuanto al RAE, ya que en la media de ambos se dio un $79 \%$ de nacidos en el primer semestre.

Otros estudios realizados en fútbol son los realizados con categorías inferiores (e.g. Barnsley, et al., 1992; Baxter-Jones, Helms, Maffull, Baines-Preece, \& Preece, 1995; Brewer, Balson \& Davis, 1995; Brewer, Balson, Davis \& Ekblom, 1992; Helsen, et al., 1998, Helsen, et al., 2000). De especial interés es el realizado por Glanser y Vincent (2004) en Estados Unidos entre los jugadores seleccionados para el programa olímpico del 2010. Este grupo de jugadores nacidos en 1984, fueron seleccionados en el año 2000, a la edad de 17 años, y estaba compuesto por 147 jugadores masculinos, de los cuales el $70 \%$ eran nacidos en el primer semestre del año, otro dato revelador encontrado en esta investigación es que entre el citado grupo había tres veces más jugadores nacidos en el primer cuarto del año que en el cuarto o cinco veces más nacidos en enero que en diciembre.

Otro estudio representativo es la investigación realizada con jóvenes futbolistas por Helsen et al. (2005) en la que analizaron a 2175 sujetos. Esta investigación se centra en analizar las selecciones nacionales de las categorías sub-21, sub-18, sub-17, sub-16 y sub-15 en la temporada 1999-2000 y los jugadores de clubes sub-14 y sub-12 participantes en dos torneos internacionales, ambos realizados en Bélgica en el año 2000. Los resultados muestran efectos significativos en las selecciones de Dinamarca, Bélgica, Inglaterra, Francia, Alemania, Italia, Holanda, España y Suecia. La mayor desproporción entre los nacidos en el primer trimestre del año y el último fue encontrada en las selecciones de Alemania, un 50.49\% en el primer trimestre frente a sólo un 3.89\% en el último; y en Inglaterra, $50 \%$ y $17 \%$ respectivamente. No existión evidencia de RAE en las selecciones de Portugal así como en la categoría sub-21 masculina y la sub-18 femenina (la única analizada). Por su otra parte, en los torneos por clubes sub-14 y sub-12 los resultados globales mostraron un $32.64 \%$ de nacidos en el primer trimestre y un $15.95 \%$ en el último.

Dentro del amplio abanico de estudios, vamos a destacar tres por el componente no sólo de constatación de RAE, sino de relación con otras variables. En un reciente estudio Augste y Lames (2011), no sólo encontraron evidencias de RAE en la categoría sub-17 del fútbol de élite alemán, sino que fueron capaces de relacionar el potencial éxito deportivo de un equipo con la fecha de nacimiento de sus jugadores, es decir, a mayor RAE, mayor provabilidad de acabar más arriba en la clasificación liguera. Igualmente reseñables son los estudios realizados por Vayens et al. (2005) y el de Helsen et al. (1998). El primero por haber analizado el RAE en un mismo contexto con diferentes fechas de corte y por aportar una profundización en cuanto al RAE, llegando al grado de implicación de los sujetos dentro del equipo y no solo a sus pertenencia a él. Y el segundo por relacionar el RAE con el abandono deportivo. Vaeyens et al. (2005) establecen dos objetivos en su estudio, por un lado comparar el RAE en los jugadores antes y después del cambio de fecha de corte que se produjo en 1997 en Bélgica, fecha en la que se pasó del 1 de agosto al 1 de enero como fecha de inicio del año de selección (estudio A). Y por otro lado usar variables relacionadas con la participación en los partidos para examinar el RAE. Estas variables son los minutos jugados y el porcentaje de partidos para los que los jugadores fueron convocados (estudio B). En el estudio A analizan la distribución en las fechas de nacimiento en 2757 jugadores de la segunda y tercera división Belga en cinco temporadas (1988-99 hasta 2002-03). Identifican dos grupos de jugadores, los nacidos antes del año 1980 (grupo A1) y nacidos posteriormente (grupo A2). Los primeros fueron determinados como seleccionados con el antiguo sistema de corte, y los segundos con el nuevo, esta distribución la basan en los estudios realizados sobre el cambio de fecha de corte por Helsen et al. (2000). Los resultados dan positivos en cuanto a una mayor presencia de jugadores nacidos en el primer cuarto del año seleccionable, sin embargo, para el grupo A1 la proporción de fechas de nacimiento disminuye casi gradualmente hacia el final del año de selección, mientras que en el grupo A2 se encuentra un gran pico en los nacimientos de agosto (primer mes del antiguo año de selección), no siendo además significativo en el grupo de los jóvenes, pese a ser mayor el número de jugadores nacidos en el primer cuarto que en el último. Otros estudios similares dieron resultados también similares (Helsen, et al., 2000).

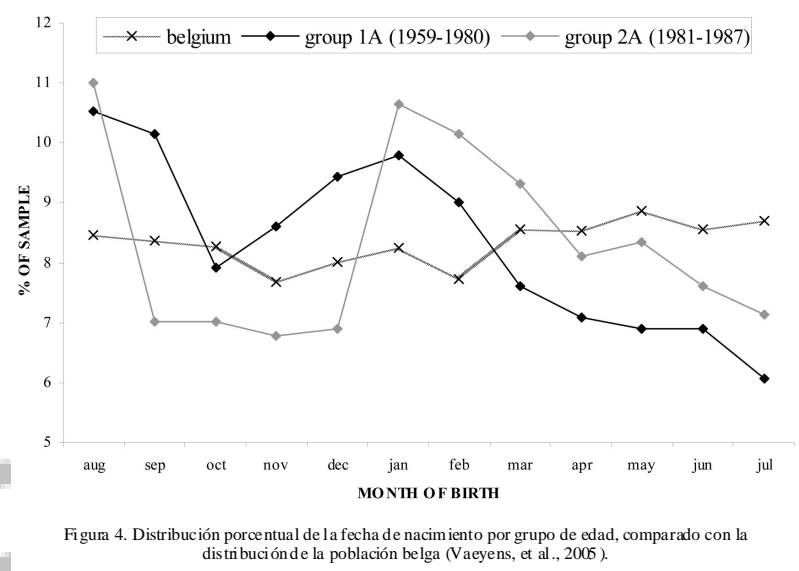

Las principales conclusiones derivadas de este estudio fueron las siguientes:

1.Debido a la persistencia de RAE después del cambio de fecha de corte, queda demostrado que la fecha de corte es la causa principal de la distribución asimétrica de la fecha de nacimiento.

2.¿Por qué los jugadores pertenecientes al grupo A1 seguían manteniendo una distribución en las fechas de nacimiento relacionada con la anterior fecha de corte? Posibles explicaciones :

a.Al ser más maduros antes del 1997 y haber promocionado a mejores equipos con mayor nivel competitivo y mejores entrenamientos, les proporcionó competencia suficiente para enfrentarse a jugadores mayores que ellos cuando cambió la fecha de corte.

b.El efecto producido por la primera fecha de corte había hecho abandonar a un porcentaje significativo de jugadores nacidos al final del año, antes de los 12 años, con lo que la proporción total era bastante favorable a los nacidos a principio del año (lo que no contemplan los autores es que la mayoría de los jugadores del grupo Al ya no competirían con los de su misma edad sino en categorías absolutas, no siendo por lo tanto importante las diferencias de maduración anuales, sino el historial deportivo).

3.En cuanto al grupo $\mathrm{A} 2$, los resultados y estudios anteriores parecen indicar que el RAE para el nuevo año de selección sería más pronunciado con el tiempo.

En el estudio B Vaeyens et al. (2005), manteniendo los grupos anteriormente descritos, analizan la distribución de las fechas de nacimiento en relación a los minutos jugados por partido y el número de

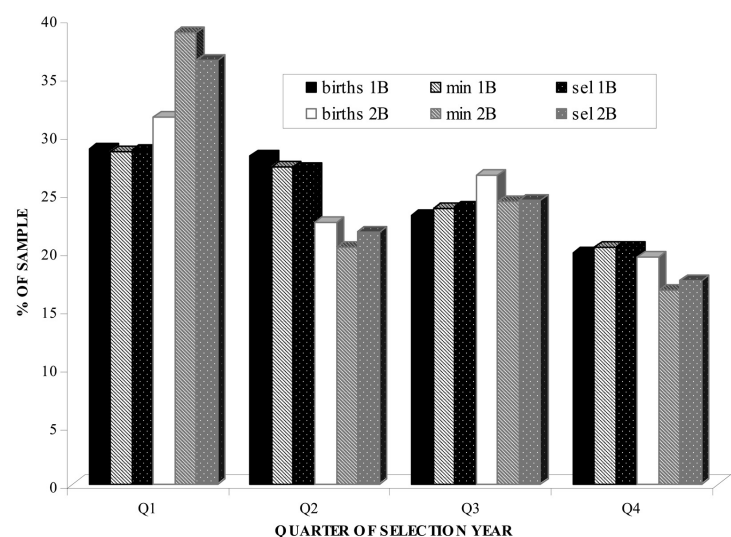

Figura 5. Distribución porcent ual por trimestre del total de veces selecionados (sel), ti empo jugado ( $\min$ ) fehca de nacimiento. Grupo 1B primer trimestre: de agosto a ocutbre. Grupo 2B: de enero a marzo 
partidos que los jugadores fueron seleccionados. En este estudio las distribuciones de los jugadores es muy similares al anterior estudio, siendo algo más pronunciada en los más jóvenes la participación activa en los partidos que el porcentaje de nacimientos. Por lo tanto, en el grupo $1 \mathrm{~B}$, una vez que se pertenecía al equipo, las oportunidades de jugar eran para todos iguales, y para el 2B seguían siendo menores para los nacidos al final del año de selección, por lo que se puede concluir que aún tenían desventajas madurativas.

Este nuevo enfoque de estudio del RAE parece más adecuado para conocer en profundidad qué pasa realmente en nuestros equipos, si bien parece en especial adecuado para categorías inferiores en las que podríamos conocer si las causas del abandono deportivo de los más jóvenes se produce realmente por una menor participación en la competición. Un estudio relacionado con estos últimos es el de Simmons y Paull (2001), quienes realizan un estudio y comparación entre Suecia e Inglaterra, ambos paises con diferentes fechas de corte. En ambos casos se analizaron categorías inferiores de jóvenes talentos y jugadores senior. La fecha de corte es el determinante de la concentración de fechas de nacimiento en todos los casos.

Debemos destacar el ya mencionado estudio de Helsen et al (1998) ya que es uno de lo más rigurosos en cuanto al análisis del RAE en las diferentes etapas de la formación por las que pasa un jugador hasta llegar a la élite. Para conocer la presencia del RAE en el proceso establecieron 4 etapas: iniciación, detección de talentos, selección de talentos y etapa final; así mismo analizaron en cada una de estas etapas la distribución en las fechas de nacimiento de jugadores belgas.

Los resultados, donde se comparan el primer trimestre del año seleccionable y el cuarto, se muestran en la siguiente tabla, en la que puede observarse una presencia masiva de RAE en todos los apartados, menos en los primeros años de iniciación. Hemos seleccionado este estudio precisamente porque es uno de los pocos que analiza las categorías inferiores, estableciendo la edad de los 12 años como la barrera en la que empieza a existir RAE, determinando por lo tanto que es a esa edad en la que se produce un abandono masivo por parte de los jóvenes futbolistas de la práctica deportiva. Resultados similares fueron encontrados con anterioridad en el Hockey por Barsley y Thompson (1988, en Helsen, et al., 1998).

\begin{tabular}{|l|l|c|c|}
\hline \multicolumn{5}{|c|}{ Tabla 3. Distribución de la fecha de nacimiento en las 4 etapas de formación del futbolista (Helsen, et al., } \\
1998).
\end{tabular}

En la siguiente gráfica se ve claramente cómo las categorías inferiores a 12 años no muestran RAE, mientras que sí lo hay a partir de los 12, según nuestra denominación de categorías, existiría discriminación a partir de la categoría infantil.

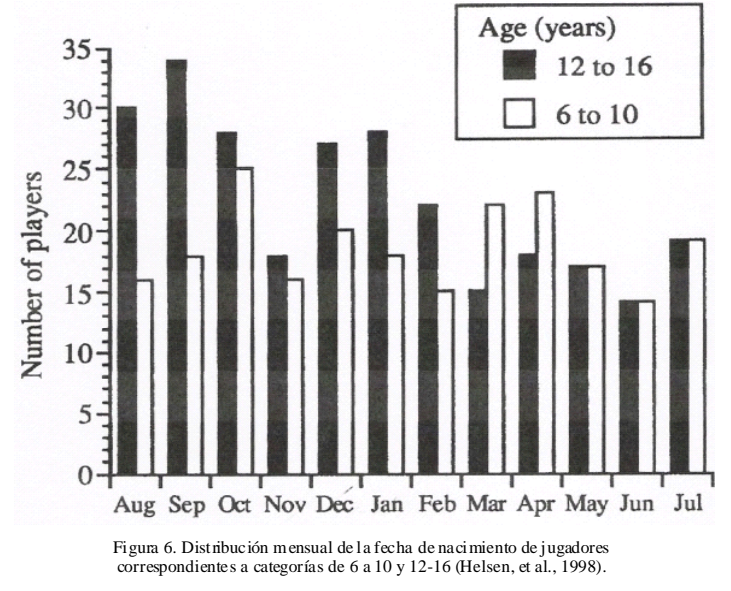

\subsubsection{Estudios sobre el RAE en el fútbol español.}

Existen diversos estudios centrados en el fútbol español así como datos sobre las selecciones nacionales españolas en distintos artículos sobre competiciones internacionales. Las primeras publicaciones donde está presente el fútbol español son aquellas en las que aparecen las selecciones nacionales al formar parte de competiciones internacionales (e.g. Barnsley, et al., 1992; Helsen, et al., 2005). Ejemplo de estos trabajos es el de Helsen et al. (2005), quienes incluyen en su estudio a las selecciones sub-16 y sub-18 en la temporada 1999-2000. En este estudio encuentran que los nacidos en el primer trimestre del año de competición eran el $45.83 \%$ con respecto al número total, frente al $6.94 \%$ de los sujetos nacidos en los tres últimos meses del año.

A partir del estudio publicado por González Aramendi (2004; estudio publicado en prensa y que posteriormente aparece como artículo en González Aramendi [2007]) sobre la incidencia del RAE en el fútbol guipozcuano, aparecen diversos estudios, unos centrado en el fútbol español en general, utilizando muestras de selecciones nacionales y clubes como el de García Álvarez y Salvadores Canedo (2005), quienes demuestran la presencia de RAE en el fútbol profesional a distintos niveles de competición y edades; en el proceso de formación de deportistas (Martín Acero et al., 2007), hasta el más reciente publicado por Lesma et al. (2011) sobre el RAE en la liga española. Varios de ellos se han centrado en las categorías inferiores. Entre estos últimos se encuentra el de Gómez Piqueras (2009), quien estudia la incidencia del RAE en la continuidad de los futbolistas desde las categorías inferiores al los primeros equipos, así como los dos artículos más importantes publicados hasta la fecha, si atendemos al nivel de las revistas donde se publican: Gutierrez Díaz del Campo et al. (2010) y Pérez Jiménez y Matthew (2008). Veamos a continuación un breve resumen de estos trabajos.

González Aramendi (2004 y 2007). En este travajo se evaluó el RAE en los equipos profesionales de fútbol guipuzcuano, así como las diferencias antropométricas y de trabajo físico entre jugadores seleccionados nacidos al comienzo y al final del año de competición. Para ello analizaron al 30\% de los jugadores federados de la temporada 2002-2003. Se encontró RAE en todas las categorías, excepto en el femenino territorial (52.3/47.7), sí dandose en el femenino nacional (61.2/38.8). Los mayores valores de RAE se dieron en juveniles masculinoy cadetes. El valor medio de RAE en cada una de las tres categorías juveniles de la liga guipuzcoana es de 66.6/33.3, 73.5/26.5 y $61.5 / 38.5$, siendo especialmente elevadoen los equipos de la Real Sociedad. No se encontraron diferencias significativas en los valores medios de los parámetros antropométricas y de trabajo físico.

García Álvarez y Salvadores Canedo (2005). En este trabajo se aborda el RAE desde una interesante perspectiva a la hora de exponer su origen y consecuencias. Se parte de un caso real comparando dos jugadores de un mismo equipo, y se cuantifican las diferencias que pueden producirse en dos sujetos debido a la edad relativa en los factores talla, peso, experiencia vital, experiencia futbolística y factores motivacionales. Los autores aportan además evidencias de RAE en distintas categorías, y selecciones, tanto del fútbol base como profesional. Los datos aportados, aunque extensos y significativos no pueden ser contrastados puesto que no explicitan las fuentes o incluso en algunos casos de las temporadas en las que fueron recogidos. La principal aportación del estudio es la propopueta para remediar el problema del $\mathrm{RAE}$, que consistiría en realizar el paso de categoría no por años, sino en el momento de la fecha de nacimiento.

Martín Acero et al. (2007); Martín Acero y Lago Peñas (2005). Estos autores realizan la reflexión de que el RAE no puede evitarse, por lo que lo importante es su incorporación como variable de primer orden en la metodología del rendimiento deportivo, esto será posible si la formación está basada en Programas Individualizados de Aprendizaje y Adaptación, mediante un enfoque sistémico y dinámico. Realizan además una comparación entre sus estudios del RAE en la LPF de las temporadas 2004/5 (datos de Martín Acero, et al.) y 2006/7. En esta comparación se constata un mantenimiento del nivel de RAE, donde aproximadamente el número de jugadores nacidos en el primer trimestre del año es el doble de los nacidos en el último. La principal aportación de 
este artículo es la descripción de un estudio de caso, en el que se describe de forma detallada el RAE en el R.C. Deportivo de la Curuña, realizando apuntes de cómo se debería realizar el seguimiento y disminución del RAE. Sin embargo no se aportan datos experimentales de ningún programa de intervención.

Gómez Piqueras (2009). Este artículo pretende analizar el RAE en las categorías inferiores de los equipos de fútbol de élite y su continuidad en el alto nivel en la temporada 2008/9. Para ello estudia el RAE en las categorías infantil, cadete y juvenil en 8 equipos de élite y en las categorías inferiores de la selección Española, además de las 8 primeras selecciones clasificadas en la Eurocopa 2008. Sin embargo, estimamos que para cumplir el objetivo planteado por el autor, el estudio debería ser longitudinal, y no transversal, por lo que la conclusión de no continuidad del RAE queda en entredicho. Los resultados muestran un marcado RAE en las categorías inferiores (74/26), pero la no existencia en el nivel profesional 50/50. Este último dato es el más destacable del estudio, por no ser coincidente con la mayoría de las investigaciones realizadas.

Pérez Jiménez y Matthew (2008). Este es un estudio sobre las categorías inferiores de la selección española sub-17 hasta la sub-21, además de considerar las categorías inferiores de clubs pertenecientes a la Liga de Fútbol Profesional española. En esta investigación se pudo constatar un marcado RAE, a favor de los jugadores nacidos en el primer trimestre del año, tanto en los equipos nacionales como en los clubs.

Gutiérrez Díaz del Campo, Pastor Vicedo, González Víllora y Contreras Jordán (2010). En este estudio se analizan las categorías inferiores (alevín, infantil, cadete y juvenil) de 20 clubes profesiones de la liga española en dos temporadas distintas (Elite 1: temporada 20052006; Elite 2: temporada 2008-2009), analizando asíla posible evolución del RAE en el tiempo. Se comparan además estos grupos con una amplia muestra de jugadores amateurs de las mismas categorías. También se analiza el RAE en función del año del jugador en su categoría, posición de juego y categoría del equipo dentro del club. Solamente los grupos de élite mostraron RAE con respecto a la población española. Se muestra una disminución del RAE entre las dos temporadas estudiadas. No se encontró influencia de las variables estudiadas en elRAE. En este estudio se hace una amplia revisión del RAE en el fútbol mundial, así como un análisis detallado de las diferentes hipótesis y teorías en torno al origen y posibilidades de intervención del RAE.

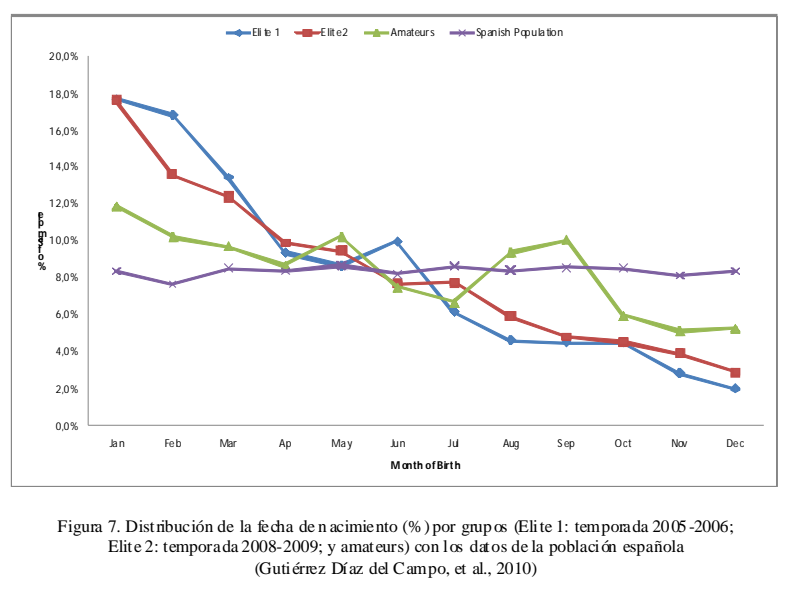

Lesma, Pérez González y Salinero (2011). Este es el más reciente estudio publicado sobre el fútbol español. En él se analiza a todos los futbolistas de la Liga BBVA de la temporada 2009-10. Los resultados muestran que un $61 \%$ de los jugadores son nacidos en el primer semestre del año. En este estudio se muestra igualmente el RAE analizado por equipos, puestos en el campo y nacionalidad. Por nacionalidad no hubo diferencias significativas, aunque el RAE entre los extranjeros es algo menor. Por puesto el mayor RAE se dio entre los defensas (64.2/35.8) especialmente cuando se realizó el análisis por trimestres $(41.97 \%$ de los defensas nacieron en el primer trimestre). El menor RAE fue encontrado entre los delanteros (55.4/44.6).

\subsubsection{Otros deportes.}

El RAE ha sido estudiado en otros deportes, siendo los resultados desiguales yen términos generales positivos aunque menos contundentes que los expuestos hasta este momento en el fútbol y hockey hielo, con la excepción del tenis y la natación donde se encuentra el mayor RAE de todos los deportes revisados. Coincide que en ambos deportes existe una gran precocidad en la alta competición. Otros deportes en los que se ha estudiado el RAE son el Beisbol (Grondin \& Koren, 2000; Stanaway \& Hines, 1995; Thomson, Barnsley \& Stebelsky, 1991; Thomson, Barnsley \& Stebelsky, 1992) donde el mayor RAE fue encontrado entre los profesionales de Japón por Grondin y Koren (2000). En baloncesto Daniel y Janssen (1987) no encontraron RAE en la NBA en la temporada 1984-1985. Esteva et al. (2006) muestran la tendencia en el baloncesto español a disminuir el RAE conforme avanzan las categorías, no encontrando RAE en la muestra de jugadores de la NBA incluidos en este estudio. Esto coincide con otros estudios realizados sobre esta liga, lo que ha venido explicándose por el tardío proceso de selección y detección que tiene este deporte en Estados Unidos, lo que parece indicar que la velocidad de desarrollo madurativo y la precocidad en los procesos de selección, así como de una especialización temprana son determinantes en la presencia de RAE.

En cricket Edwards (1994) analizó las fechas de nacimiento de los jugadores de la liga entre condados y encontró un RAE muy dependiente de la posición del jugador, esta variación iba del $61 \%$ de nacidos en el primer semestre, a no existir RAE en otras posiciones. En fútbol americano también existen diversos estudios (e.g. Daniel \& Janssen, 1987: Glamser \& Marciani, 1992; Stanaway \& Hines, 1995). El realizado con profesionales de este deporte en Estados Unidos y Canadá por Daniel y Janssen (1987) no evidenciaron RAE. En gimnasia (BaxterJones, et al., 1995) encontraron poca evidencia de RAE. En balonmano encontramos el estudio realizado por Ryan (1989) con categorías comprendidas entre los 11 y 20 años, donde se constataron grandes diferencias en el RAE dependiendo de la categoría y el nivel de competición. En natación Baxter-Jones (1995) evidenciaron una gran desproporción en las fechas de nacimiento entre los nadadores de élite junior ingleses; casi tres cuartas partes de ellos eran nacidos en la primera mitad del año competitivo. En este mismo deporte Ryan (1989)encontró grandes diferencias dependiendo de la categoría y el género.

En tenis los estudios han sido realizados por debajo de los 18 años (Baxter-Jones, 1995; Dudink, 1994; Edgar \& O'Donoghue 2005; Giacomini, 1999), evidenciando una fuerte influencia del RAE, sin embargo algunos de los estudios realizados con muestra femenica han dado resultados negativos en cuanto al RAE (Giacomini, 1999; Ryan, 1989). En voleibol Grondin et al. (1984) encontraron menor RAE en el deporte femenino y una decreciente RAE con el aumento de la edad. Similares resultados arrojó el estudio de Ryan (1989). En un reciente estudio Medic, Young, Starkes, Weir y Grove (2009) encontraron evidencias de RAE en la participación y en el éxito de nadadores y atletas de élite estadounidenses, siendo similar en ambos deportes, y más acusado en hombres que en mujeres. El dato más interesante aportado por este estudio es que el RAE aumenta con la edad de los deportistas, algo que ocurre en el sentido inverso en otros deportes como el baloncesto, voleibol o fútbol, tal y como hemos visto anteriormente. Nakata y Sakamoto (2011) realizan un estudio de deportistas de élite japoneses de diversos deportes, encontrando evidencias de RAE en la mayoría de ellos, aunque no en todos.

\subsubsection{Deporte femenino.}

La mayoría de los estudios realizados sobre elRAE han contemplado únicamente a deportistas masculinos, pero aquellos en los que se ha incluido al deporte femenino (e.g. Giacomini, 1999; Grondin, et al. 1984; Helsen, et al. 2005; Ryan, 1989) han mostrado, con independencia del deporte, de forma clara, una menor presencia o incluso ausencia del RAE que en sus homólogos masculinos. Sin embargo debemos tener en cuenta recientes estudios como el realizado por Delorme, Boich y Raspaud (2010), quienes advierten del poco estudio del RAE en el 
deporte femenino, además de mostrar su presencia en el futbol estadounidense femenino, tanto en la composición de los equipos de élite como en el abandono deportivo. Precisamente por la poca presencia de RAE establecida en la mayoría de los estudios, es importante hacer un especial seguimiento del RAE en el deporte femenino para ver si, en caso de aparecer como en el estudio mencionado, intentar determinar los elementos que lo hayan hecho aparecer en deportes donde previamente no existía.

\subsubsection{Abandono deportivo y adhesión a largo plazo.}

Anteriormente se han expuesto los estudios que relacionan el RAE y abandono deportivo. Destacando los anteriormente ampliados estudios de Barsley y Thompson (1988), Helsen et al (1998) y Delorme, Boich y Raspaud (2010). Al haber sido el RAE tradicionalmente estudiada en deportistas en activo, el estudio de Schorer, Neumann, Cobley y Baker (2011) aporta nuevos datos al centrarse en el efecto de la edad relativa en la involucración a largo plazo en el deporte. Estos autores muestran la existencia de RAE en las carreras de antiguos jugadores de baloncesto, que siguieron ligados al mundo deportivo con roles como los de árbitro o entrenador, indicando la posibilidad de que los efectos durante su carrera como deportistas, tuviesen un efecto prolongado en el mundo deportivo, más allá de la carrera como jugadores.

\subsubsection{RAE y condición física.}

Roberts, Boddy, Fairclough y Stratton (2012) en un reciente estudio en el que analizaron los datos de condición física cardiorespiratoria, extraidos de una gran base de datos (11404 niños de 9-10 años y 3911 niños de 11-12 años), con el mes de nacimiento. Los resultados muestran una relación signigicativa del mes de nacimiento y los parámetros medidos ( $<.01$ ), incluso cuando se realizó un control de maduración somática (p<.05). Un estudio que no aborda el RAE directamente, pero que está muy relacionado es el de Portella, de Arruda y Cossio-Bolaños (2011) quienes realizan una valoración del rendimiento físico en jóvenes futbolistas entre 11 y 18 años en función de la edad cronológica.

Una vez expuesto el estado de la cuestión abordaremos y clasificaremos los principales motivos causales del RAE, así como las soluciones propuestas desde el ámbito científico para mitigar sus efectos negativos.

\subsection{Hipótesis Explicativas y Potenciadores del RAE}

\subsubsection{Hipótesis madurativa.}

Centraremos a continuación nuestra revisión bibliográfica en las hipótesis planteadas para explicar el RAE, siendo la hipótesis madurativa la más extendida. Esta hipótesis se apoya en las potenciales diferencias madurativas debidas a la diferencia de edad cronológica dentro de un mismo curso o categoría. Estas diferencias pueden amplificarse cuando entran en juego los distintos ritmos madurativos. El caso más evidente sería la comparación de un sujeto nacido al principio del año de selección y que sea prematuro en su maduración, frente al caso contrario de otro sujeto nacido al final del año de selección y tardío en cuanto a su maduración.

Las diferencias cronológicas entre sujetos que componen un mismo grupo pueden derivar en diferencias en las capacidades asociadas a los procesos madurativos. En este sentido Martin, Foels, Clanton y Moon (2004) afirman que la maduración neurológica puede manifestarse en distintas capacidades tales como la de autorregulación de la atención, la emoción y otras funciones como la memoria (Siegler, 1991), en especial aquellas localizadas en los lóbulos frontales. Estas funciones incluyen la atención selectiva (Miller, 1991) algunos aspectos de la metacognición (Garner, 1991) y del control de inhibición (Barkley, 1998). En cuanto a las medidas antropométricas, y capacidades condicionales, existen numerosos estudios que demuestran que su desarrollo está asociado a la maduración, y ésta, condicionada por la edad. Malina, Bouchard, BarOr(2004) y Malina (1994) realizan una magníficarecopilación de estudios donde se establecen los ritmos y cronología de las modificaciones corporales y motrices, siendo estas obras los principales referentes de aquellos autores que utilizan la ventaja madurativa como explicación del RAE. Asimismo en Malina (2003) se puede consultar de forma más específica las implicaciones del crecimiento y maduración en la selección y rendimiento de jugadores de fútbol. De forma similar se aborda el tema de la predisposición para ser seleccionado en fútbol según las características antropométricas y fisiológicas en Reilly, Bangsbo y Franks (2000). Tema presente también en la obra de Ward y Williams (2005) sobre la naturaleza multidimensional de la expertise en fútbol, aunque en este caso se centran en el desarrollo de las capacidades congnitivas y perceptivas.

\subsubsection{Hipótesis del autoconcepto.}

La segunda hipótesis es denominada hipótesis del autoconcepto (Thompson et al., 2004). Esta hipótesis sostiene que el efecto acumulado por las desventajas madurativas hace que disminuya la autoestima del sujeto, por lo que se involucraría menos en la actividad escolar y lograría peores resultados (Pellegrini, 1992). El efecto sería el contrario para aquellos que reciben desde un inicio, refuerzos positivos por sus ventajas madurativas. Estas explicaciones, aunque estudiadas en diversas ocasiones, no han recibido suficiente soporte empírico hasta el momento (Bickel, et al., 1991; Spitzer, et al., 1995).

\subsubsection{Hipótesis de la experiencia.}

Unidos a los factores físicos y psicológicos en los que los niños nacidos en la primera parte del año de competición tienen potencialmente ventaja sobre el resto, está el factor de la experiencia, siendo ésta la tercera hipótesis. Para un niño de 10 años, la diferencia de tiempo vivido con respecto a un compañero al que le adelanta en casi un año de vida es de un $10 \%$ más, lo cual se puede convertir en casi un año de experiencia en el deporte en concreto. Los efectos de la acumulación de este entrenamiento aumentan las posibilidades de participación activa en los distintos deportes. Esta diferencia de entrenamiento puede estar magnificada si tenemos en cuenta que los niños mayores tienen mayor probabilidad de ser elegidos para formar parte de los equipos o de jugar más tiempo durante la competición. Esta selección no está únicamente asociada a recibir mejores entrenamientos, sino también a aumentar la motivación yla probabilidad de seguirla práctica deldeporte sin abandonar.

3.3.4. Agrupación por nivel de rendimiento temprana y acceso a categorías superiores.

Una agrupación por nivel de rendimiento temprana (earlystreaming) y el sistema de acceso a categorías superiores (slotting system), en el caso del fútbol cada dos años, son los dos factores que establecen tanto Daniel y Janseen (1987) y Thompson y Barnsley (1985, en Hurley, et al., 2001) como necesarios para la aparición de RAE. Esta hipótesis es válida para los deportes como el fútbol o el hockey en su versión masculina, pero no explica la existencia o ausencia de RAE en otro tipo de deportes y su ausencia en el deporte femenino.

\subsubsection{Hipótesis de la influencia paterna en el enrolamiento} inicial

Dos recientes estudios con jugadores menores de siete años muestran la existencia de RAE en contextos deportivos con estructuras no competitivas, donde no existe selección previa por parte de la institución deportiva. Delorme, Boiché, y Raspaud (2010) estudian a jugadores de fútbol franceses menores de siete años, y Hancock, Ste-Marie, y Young, (en prensa) examinan la distribución en jugadores de hockey canadienses de 5-6 años. En ambos casos existió una sobrerepresentación de aquellos relativamente mayores. Igualmente en ambos casos se sugiere que existió un sesgo en el enrolamiento, siendo altamente probable que este sesgo fuese debido a la acción de los padres, puesto que la estructura deportiva en uno y otro contexto eran no competitivas. Esta hipótesis por lo tanto plantea que la acción paterna sería determinante en el origen del RAE, cuando se da en aquellas edades que por ser muy tempranas, su opinión y acción es determinante a la hora de incluir al niño en actividades deportivas regladas. El sesgo paterno vendría por atribuir a los niños relativamente mayores unas mayores expectativas de éxito, basadas 
éstas en la comparación con su grupo de iguales, entre los que destacarían por su ventaja cronológica.

\subsubsection{Potenciadores del RAE.}

Aparte de las hipótesis expuestas, existen una serie de factores que han sido descritos como potenciadores del RAE (Musch, 2001):

El nivel de competición. Se ha verificado una mayor presencia de RAE cuanto mayor es el nivel de competición. Aunque hay poca bibliografía al respecto de participación en deporte recreativo, parece evidente que cuanto mayores sean los filtrados derivados de los procesos de selección, y así ocurre cuanto mayor es el nivel de competición, mayor será el RAE. Se confirma este factor en estudios como el que se ha expuesto anteriormente de Barsley y Thomson et al. (1988) sobre el hockey hielo, o el de Gutiérrez Díaz del Campo et al. (2010) donde las escuelas deportivas mostraron menor RAE que los equipos de élite de las mismas categorías.

La popularidad del deporte. Se ha constatado mayor RAE en los deportes mayoritarios, debido a que los más competentes, entre los que un significativo porcentaje lo son por un mayor desarrollo debido al $\mathrm{RAE}$, se derivan naturalmente y de forma prematura hacia los deportes de mayor prestigio.

El sistema de categorías cada dos años. La existencia de sujetos dentro de un rango de edad de 2 años hace que las diferencias intracategoría sean mayores.

Especialización temprana. En aquellos deportes en los que se dan procesos de selección tempranos aumenta la probabilidad de que los menos maduros y con menos experiencia queden fuera, sin posibilidad de incorporarse a procesos de entrenamiento que por lo general son altamente especializados y restringidos

Expectativas de aquellos encargados de evaluar y seleccionar. Una teoría muy estudiada en el ámbito pedagógico, como es el Efecto Pigmaleón, puede explicar el RAE. Esta teoría, que argumenta que las expectativas sobre las capacidades que de un alumno tienen padres, educadores y compañeros son traducidas en comportamientos explícitos e implícitos, lo cual influye en el rendimiento real del alumno. El efecto Pigmaleón puede amplificar y estabilizar las desventajas derivadas de las diferencia de edad dentro de un mismo grupo (Rejeski, Darracott \& Hutslar, 1979; Landers \& Fine, 1996).

Fecha de inicio del año escolar. Este factor haría que no solamente el RAE se debiera a los procesos ocurridos dentro del sistema deportivo, sino que la más que demostrada presencia de RAE en el ámbito escolar, los potenciase. Este factor solo podrá demostrarse en países y deportes en los que la fecha de inicio del año escolar sea distinta a la del año competitivo. Vayens et al. (2005) dan como no válida o de poca importancia la fecha de corte del sistema educativo, ya que en sus estudios realizados en Bélgica sobre la presencia de RAE después de 1997, cuando se cambió la fecha de corte en el fútbol federado, con lo que dejó de coincidir con la fecha de corte escolar, los alumnos nacidos al principio del curso escolar (1 de agosto) reducían su presencia en los equipos de fútbol, si bien seguía existiendo un significativo efecto residual que no es explicado enteramente por los autores y que sí podría validar esta suposición.

- Otros. Musch y Hay (1999) establecen la existencia de otros factores que podrían influir en el nivel de RAE encontrado en las distintas investigaciones, tales como la estación de nacimiento, condiciones socioeconómicas o los factores climáticos, aunque los desechan al realizar estudios internacionales, donde dichas condiciones eran distintas.

\subsubsection{Modelo ecléctico para explicar el RAE en el deporte.}

Para acabar el apartado dedicado a las hipótesis explicativas del RAE queremos exponer la teoría manejada por muchos autores y expuesta explícitamente por Thompson et al. (2004), quienes poseen varias publicaciones relacionando los distintos ámbitos en los que se ha encontrado RAE. Para estos autores, la cadena que enlaza la fecha de nacimiento con los logros escolares y deportivos, autoestima y suicidio contiene los siguientes pasos:
1. La edad relativa produce diferencias en los logros debido a la maduración, no a la capacidad.

2. Estas diferencias en los logros y éxitos llevan a variaciones en la autoestima y confianza.

3. La baja autoestima y la falta de autoconfianza están asociados con la incapacidad infantil para competir con sus compañeros de grupo, conduciendo respectivamente a la depresión y desesperanza. En último extremo, la depresión y desesperanza adquirida, los cuales son generalmente considerados como los ingredientes esenciales del suicidio, se convierten en los precursores de comportamientos autolesivos.

Las hipótesis expuestas, al menos por separado, no permiten explicar completamente cómo «ventajas madurativas en las primeras edades llevan a logros deportivos a largo plazo» (Baker et al., 2010, p. 27). Es por tanto, altamente probable que todas ellas tengan una importancia relativa en los distintos casos de RAE en el deporte, siendo su importancia y relación entre ellas distinta en función del caso individual.

A continuación intentaremos trazar el papel de cada una de las hipótesis en un caso ficticio, para entender así cómo pueden interrelacionarse y reforzase a lo largo del tiempo. Imaginemos a Raúl, un niño nacido en enero que desde la escuela infantil ha destacado entre sus compañeros nacidos en la segunda parte del año. Juan, estimulado por su entorno correctamente, alcanza una eficacia alta en los patrones motrices mucho antes que sus compañeros. Sus padres, viendo cómo es capaz de botar una pelota, conducir con el pie un balón o golpear eficazmente una bola con una raqueta antes de los cinco años, deciden apuntarlo a un club deportivo. Partiendo de este caso inicial, cuando un niño (Juan en este caso) se enrola de forma muy temprana en una institución deportiva, el papel paterno es determinante (hipótesis del enrolamiento temprano). Asu vez, la percepción paterna vendrá originada por las ventajas madurativas (hipótesis madurativa). Si Juan se mantiene en el sistema, avanzando por los distintos niveles que exigen cada vez más compromiso, entrará en juego la motivación intrínseca, relacionada con la autopercepción (hipótesis del autoconcepto). Dentro del sistema deportivo, si existen pruebas de selección para establecer categorías por edades, o por niveles (hipótesis de agrupación por nivel de rendimiento temprana y acceso a categorías superiores), especialmente si son en las primeras categorías, la ventaja de Juan, nacido en las primeras fechas del año serán aún las madurativas, pero también sumada las de la experiencia (hipótesis de la experiencia). En los casos, como el expuesto, en los que el niño se beneficie además de un enrolamiento temprano, la experiencia será aún mayor que la que le otorga su mayor edad cronológica. A todos estos efectos debemos sumar la idea general de que aquel que entra en el sistema primero, entendiendo por sistema una institución deportiva que aporta los medios suficientes para mejorar el rendimiento a corto y largo plazo del deportista, tendrá más oportunidades de triunfar, especialmente en pruebas selectivas, y por lo tanto, mantenerse en el sistema. Esta última conclusión es conflictiva, por cuanto podría entenderse como un sustento a la idea de una especialización precoz.

\subsection{Propuestas de Intervención}

Un vez que hemos revisado las principales investigaciones sobre el efecto de la edad relativa, y que ha quedado evidenciado que es la hipótesis de la maduración y la estructura competitiva la principal causa de la irregular distribución de jugadoresen cuanto a su fecha de nacimiento, vamos a enumerar soluciones propuestas por los distintos autores (García Álvarez \& Salvadores Canedo, 2005; Gutiérrez Díaz del Campo et al., 2010; Horn \& Okumura, 2011; Hurley et al., 2001), así como otras propias, para atajar este problema y hacer el sistema de competición más justo.

3.4.1. Propuestas basadas en el cambio de estructura de la competición.

Cambio en la fecha de corte: en 1995 en Estados Unidos, se cambió la fecha del 1 de enero al 1 de julio con la intención de atajar este problema, sin embargo esto sólo cambió los meses más favorables hacia el segundo semestre del año. De mismo modo fue constatado por Helsen et al. (2000) y Vaeyens et al. (2005) en el fútbol belga. 
- Retraso del proceso de agrupación por rendimiento: esta propuesta si bien no ha sido llevada a cabo por ningún organismo oficial, parece ser bastante coherente, ya que en los deportes en los que el proceso de selección se retrasa más allá de los 13 ó 14 años, tales como el baloncesto o fútbol americano, ambos en Estados Unidos, el RAE es mucho menor. Sin embargo esa política bastante perseguida principalmente por quienes nos consideramos formadores no es compartida por la mayoría de los responsables federativos.

-Retraso de la especialización deportiva: propuestas en las que se proponen aproximaciones multidisciplinares podría ser una de las llaves para retrasar la especialización y por lo tanto para realizar un enfoque más recreativo y formativo en las primeras edades, donde las diferencias son mayores. Esta estructuración deportiva no va en contra de la capacitación futura de los jugadores, sino todo lo contrario, sobre todo en aquellos deportes que pueden ser agrupados mediante un enfoque táctico. Esta propuesta mitigaría alguno de los factores potenciadores delRAE, tales como la experiencia en un deporte concreto o la derivación de los más maduros a deportes de mayor prestigio. Si además se aporta un enfoque educativo por encima del competitivo se podría disminuir el abandono deportivo. Ejemplo de la puesta en práctica de esta propuesta es la organización del deporte en edad escolar en el Pais Vasco.

- Grupos de edad más pequeños: en gran número de los artículos revisados se propone una reducción del rango de edad por categoría, esta propuesta está apoyada por resultados hallados en aquellos deportes en los que existen, según la competición, categorías bianuales y anuales, como es el caso del Hockey en Canadá. La propuesta real sería reducir a un año todas las categorías y a ser posible a 6 meses. Sólo tenemos constancia de una experiencia realizada con categorías semestrales, la registrada por Boulton (2001, en Glamser y Vincent, 2004), pero los resultados no fueron concluyentes, y los mismos autores proponen que se necesitaría másevidencias empíricas para establecersecomo un remedio al RAE. Parece que esta puede ser una buena solución a adoptar de forma interna dentro de aquellos clubes preocupados por la cantera y conscientes de este problema.

Cuotas: algunos autores han aconsejado establecer cuotas o porcentajes de jugadores nacidos en cada uno de los semestres del año. De esta forma se aseguraría la participación por igual de todos los jugadores. Esta propuesta es criticada por Hurley et al. (2001), ya que según estos autores ello magnificaría el RAE, más allá de mitigarlo. Bajo nuestro punto de vista, esta medida sólo sería eficaz si se estableciera un sistema de competición en el que la participación de todos los jugadores fuese obligatoria, de otra forma lo que ocasionaría sería un gran desequilibrio en la participación activa de los jugadores, que como hemos visto, es otro efecto de la edad relativa.

-Categoría por peso o talla: establecer un límite de peso o altura dentro de cada categoría. Esta solución teóricamente reduciría el RAE ya que existe una relación directa entre el desarrollo y la talla, sin embargo las diferencias individuales en cuanto a la talla final hacen poco realista esta medida. Poco viable sería la agrupación en función del peso en deportes de equipo, pudiendo además ocurrir que los jugadores establecieran procesos de adelgazamiento para entrar con ventaja en los equipos de menor peso. En cuanto a organización competitiva la agrupación por peso se da en deportes de lucha. El estudio del RAE en estos deportes podría aportar datos sobre la conveniencia o no de extender estas agrupaciones a otros deportes individuales, dentro de las etapas de formación.

- Calendario rotativo de fechas de corte: esta propuesta se basa en que a lo largo de los años un mismo jugador no sea siempre el más joven o el mayor de su categoría. Esta fue una de las recomendaciones enunciadas en 1999 por la Asociación de Hockey Canadiense para mejorar el desarrollo del hockey en ese país. Al hilo de tal recomendación Hurley et al. (2001) propusieron un sistema para llevar a cabo esta propuesta, si bien hasta la fecha no ha sido llevada a la práctica. En su sistema se establecería un ciclo de ocho años en los que la fecha de corte iría avanzado un trimestre al año, hasta completar los 8 que posee cada categoría, de esta forma un jugadorque en la temporada 2000 perteneciera al trimestre de los mayores, en el 2003 y 2004 sería el más joven y volvería a ser el mayor en el 2007. Los autores establecen ocho razones por las que estiman que su sistema es mejor que el actual. Seis de estas razones se refieren a la facilidad de implementación y mayor justicia del sistema, una al mejor aprovechamiento de los campos de hielo (la única exclusiva del hockey hielo), y una octava relativa a la formación de los jugadores, razón más que interesante pero no demostrada: argumentan que el hecho de que cada temporada los jugadores cambiasen de equipo y de entrenadores, sería un factor beneficioso para la formación del deportista.

-División de los jugadores por su rendimiento: este enunciado es propuesto por algunos autores como una solución, argumentando que de esta forma los sujetos jugarían en igualdad de condiciones y no se produciría abandono de la práctica deportiva. Sin embargo, es establecido por otros como uno de los causantes del RAE, no solo en el ámbito deportivo, sino el escolar también. Kaiserman (2005) propone esta solución para ligas recreativas, donde el objetivo principal sea una participación satisfactoria y no el rendimiento.

- Establecer el paso de categoría en función al cumplimiento del año cronológico del jugador: esta propuesta implica que cuando el niño cumpla los años correspondientes a su categoría pasaría automáticamente de división. A favor de esta propuesta tiene los beneficios mostrados en el cambio rotativo del fecha de corte, pero una gran complejidad organizativa, tal y como exponen García Álvarez y Salvadores Canedo (2005), autores de esta propuesta.

- Diseñar las pruebas de selección (tryouts) de forma adecuada: formato de juego reducido y agrupaciones por trimestres: Horn y Okumura (2011) en un reciente trabajo, exponen varias opciones para reducir el RAE en el fútbol en Estados Unidos. Tres de sus cuatro propuestas están incluidas entre las expuestas anteriormente. La que no lo está, está referida a las pruebas de selección, donde se decide en gran medida el futuro de los jugadores. Estos autores proponen diseñar pruebas donde no prime el desarrollo físico, y donde se pueda poner en evidencia la calidad de cada uno de los jugadores. Para ello proponen desterrar formatos como el 8 vs 8 , y sustituirlos por otros más reducidos, por ejemplo el 3 vs 3, dando mayor oportunidad de participación. Es especialmente interesante esta propuesta si se combina con una agrupación de jugadores para los tryouts, por semestres o cuatrimestres, de tal forma que los jugadores presentes en los partidos de evaluación sean todos de muy parecida edad.

\subsubsection{Propuestas basadas en el funcionamiento interno de} los clubes.

La estructura del sistema competitivo es muy difícil de cambiarse, por lo tanto las soluciones propuestas en el apartado anterior están lejos de convertirse en realidad. Sin embargo, los responsables deportivos de clubes y escuelas deportivas sí que podrían de forma más factible aplicar algunas de estas soluciones, tales como la agrupación de equipos por semestres, o disminuir la presión que ejercen los resultados en la participación de los jugadores en la competición.

No se han difundido de forma sistemática, pero nos consta que algunos clubes, conscientes del problema delRAE, han decidido estudiarlo y atajarlo en la medida de lo posible. En España conocemos los casos de dos de los clubes que más cuidan su cantera, la Real Sociedad y El Athletic Club de Bilbao. Ejemplos de esta preocupación son las palabras de responsables médicos de la Real Sociedad, el doctor González Aramendi (2004), quien escribe como resumen de su artículo sobre el efecto de la edad relativa en el fútbol guipuzcuano: «en todo caso, y al margen de cualquier reforma competitiva, la llave que abre la solución está en la vigilancia capacitada y responsable de los entrenadores, preparadores, médicos, directivos de clubes y federaciones y responsables políiticos». Así como en las palabras del Doctor Angulo (2006) del Ath. Club de Bilbao : «a los más pequeños los cuidamos muchísimo, y los entrenadores saben que tienen que cuidarlos », pero también añadía : «el problema es que ya nos vienen así al club» en referencia a la desproporción de las fechas de nacimiento, y situando el problema en el fútbol base y en los procesos de selección y no dentro del funcionamiento del club, con lo cual parece que más allá de seguir 
investigando y confirmando la presencia de RAE en el deporte de competición, deberíamos preocuparnos de su origen en el deporte recreativo y en los procesos de detección que llevan a los jugadores a los grandes clubes. Es por ello que los responsables de las escuelas deportivas y el deporte escolar parecen ser los mejores situados para atajar este problema.

\section{Conclusiones}

El gran reto que se nos plantea como gestores, directivos, entrenadores y educadores es que todos aquellos ciudadanos que se acerquen al ámbito deportivo lo hagan en igualdad de condiciones, tanto si persiguen un objetivo recreativo, educativo como profesional. Si esto se consiguiera, se aumentaría la participación voluntaria en el deporte escolar y el grado de satisfacción y beneficios obtenidos por los practicantes. Se mejorarían los procesos de detección, selección y formación de elites deportivas y como consecuencia, en elúltimo escalón competitivo, se mejorarían los rendimientos de los equipos de élite, clubes y selecciones. Esto se lograría evitando aquellas consecuencias negativas que configuran el RAE para un gran número de la población: la poca adhesión a la práctica deportiva por experiencias negativas, el abandono prematuro por falta de logros o refuerzos positivos; y procesos de detección y selección que muestran graves deficiencias a largo plazo al no incluir de forma proporcional a toda la población, sino de forma muy sesgada a los madurativamente más avanzados. Todo esto pasa por considerar el RAE de nuestros alumnos y jugadores, no sólo como un tema de estudio científico, sino como un factor más dentro del proceso de formación deportiva y educativo; y porque las soluciones propuestas no se queden en una mera descripción de intenciones, sino que pasen a formar parte de los principios metodológicos utilizados en nuestra labor formativa y gestora diaria.

Hasta hoy ninguna de las propuestas ha llegado a demostrar resultados suficientes para que las instituciones tomen las medidas que se han venido planteando desde el ámbito científico, principalmente por la falta de programas de intervención serios. El problema radica en que para que se pudiera demostrar su eficacia debería haber una firme concienciación y colaboración por parte de tales instituciones. Debemos por lo tanto partir del trabajo diario y de nuestra responsabilidad para con nuestros jugadores y alumnos, vengan estos tanto a realizar deporte por salud, recreación o buscando un rendimiento y una carrera profesional. Responsabilidad que debe conllevar ofrecer las mismas oportunidades a todos ellos, sin que el rendimiento a corto plazo sea el culpable de limitar las oportunidades de formación e incluso del abandono de la práctica deportiva.

Aunque demostrada la existencia del RAE en el deporte así como en otros ámbitos, los estudios existentes han puesto de manifiesto que existen grandes diferencias según las variables contextuales, como la categoría, el deporte, el puesto dentro del equipo, el nivel de competición, el sexo, la raza o el país. Y será el conocimiento de estas variables lo que posibilitará el desarrollar soluciones eficaces y medidas de intervención reales y adecuadas. Por lo tanto, cuando nos acerquemos a una institución deportiva, este estudio debe formar parte de forma natural de nuestra evaluación inicial, tanto como el contextomaterial en el que nos movemos o el rendimiento en resistencia o técnica de regate de los jugadores de nuestro equipo. Es por ello que proponemos a todos aquellos responsables de equipos, clubes o escuelas deportivas una toma de conciencia mediante una evolución inicial de la distribución de las fechas de nacimiento de los jugadores que componen sus equipos, conocimiento en profundidad mediante el aislamiento de las variables que concurren dentro de un deporte: procedencia del jugador, posición en la que juega, número de convocatorias y minutos jugados, etc. y aplicación de soluciones adecuadas al contexto. Tales medidas deben incluir formación y concienciación sobre todo el personal de la institución: entrenadores, preparadores físicos, ojeadores, directivos e incluso sobre los mismos jugadores tradicionalmente perjudicados, que deberían ser informados sobre su valía más allá de la comparación diaria e injusta con el resto de sus compañeros mayores. Informándoles de que su total potencialidad como jugador no será evidente hasta finalizado su proceso de maduración, por lo que deben de tener una especial capacidad de compromiso a largo plazo para consigo mismos. Estas consideraciones con el jugador deben hacerse extensivas a los padres, puesto que estamos hablando de jugadores de corta edad. Por lo tanto, la aproximación al problema dentro de un club u otra institución deportiva debe fundamentarse en una política responsable y estable que emane del director deportivo y poseer un enfoque multidisciplinar que implique a entrenadores, preparadores físicos, psicólogos, etc. y cuyos destinatarios sean todos los agentes del sistema: personal del club, jugadores y padres.

En este sentido, en los últimos años se atisba una mayor concienciación sobre este problema, si bien aún no se ha plasmado en programas concretos de intervención. En este sentido diferentes programas sobre la concienciación, y en menor medida intervención, sobre el RAE están apareciendo. Ejemplos son los ejemplos de proyectos los encontramos en Gran Bretaña, en la universidad de Loughborough ${ }^{2}$, y en el South West Talent Development Centre. De igual forma en nuestro pais empieza a cobrar importancia el RAE en la formación de profesionales. Ejemplo de esto último es la introducción de ponencias específicas en el máster universitario en Detección y Formación Del Talento En Jóvenes Futbolistas ${ }^{3}$ (Gutiérrez Díaz del Campo, 2007) organizado por la Federación Española de Fútbol y la universidad de Castilla-la Mancha y en el curso de Experto Director Deportivo ${ }^{4}$ (Gutiérrez Díaz del Campo, 2011) organizado por la Liga Profesional de Fútbol y las universidades de Castilla-La Mancha y Oviedo.

Casi todo lo dicho para el ámbito deportivo puede ser transferido al ámbito escolar, donde desde el docente hasta el director del centro educativo deberían tener una especial sensibilidad acerca de este tema, prestando atención a la concienciación y formación del equipo docente, así como evaluaciones periódicas para que el RAE sea un factor más a tener en cuentaen la aplicación de medidas educativas de individualización.

\section{Referencias}

Angulo, F. (2006). Evaluación de los factores fisiológicos en el proceso de 7 selección de jóvenes futbolistas. Ponencia inédita en el Máster Universitario en Detección y Formación del Talento en Jóvenes Futbolistas organizado por la Federación Española de Fútbol y la universidad de Castilla-la Mancha. Madrid.

Augste, C., \& Lames, M. (2011). The relative age effect and success in German elite U-17 soccer teams. Journal of Sports Sciences, 29(9), 983-987.

Baker, J., Schorer, J., \& Cobley, S. (2010). Relative age effects: An inevitable consequence of elite sport? German Journal of Sport Sciences (Sportwissenschaft), 1, 26-30.

Barkley, R. A. (1998). Attention-deficit hyperactivity disorder: A handbook for diagnosis and treatment. New York: Guilford Press.

Barnsley, R. H., Thompson, A. H., \& Legault, P. (1992): Family planning: football style. The relative age effect in football. International Review for the Sociology of Sport 27(1), 77-88.

Barnsley, R. H., \& Thompson, A. H. (1988). Birthdate and success in minor hockey: The key to the NHL. Canadian Journal of Behavioural Science, 20, 167-176.

Barnsley, R.H., Thompson, A.H., \& Barnsley, P.E. (1985): Hockey success and birthdate: the relative age effect. CAHPER/ACSEPL journal (Vanier) 51(8), Nov/Dec, 23-28.

Bäumler, G. (2000): The relative age effect in soccer players and its correlation with the chronological age (Zum Zusammenhang des Relativalterseffekts mit dem chronologischen Alter bei professionellen Fussballspielern.). Sportonomics (Munich) 6(1), Mar, 25-30.

Baxter-Jones, A., Helms, P., Maffull, N., Baines-Preece, J., \& Preece, M. (1995). Growth and development of male gymnasts, swimmers, soccer and tennis players: A longitudinal study. Annals of Human Biology, 22, 381-394.

Bedard, K., \& Dhuey, E. (2006). The persistence of early childhood maturity: International evidence of long-run age effects. Quarterly Journal of Economics, 121(4), 1437-1472.

Bell, J. F., \& Daniel, S. (1990): Are summer-born children disadvantaged? The birthdate effect in education. Oxford Reviw of Education, 16, 67-80. 
Bell, J. F., Massey, A., \& Dexter, T. (1997). Birthdate and ratings of sporting achievement: analysis of physical education GCSE results. European journal of physical education, 2(2), 160-166.

Bickel, D. D., Zigmond, N., \& Strayhorn, J. (1991). Chronological age at entrance to first grade: Effects on elementary school success. Early Childhood Research Quarterly, 6, 105-117.

Boucher, J., \& Halliwell, W. (1991). The novem system: A practical solution to age grouping. Canadian Association for Health, Physical Education, and Recreation, 57, 16-20.

Boucher, J., \& Mutimer, B. (1994). The relative age phenomenon in sport: A replication and extension with ice-hockey players. Research Quarterly for Exercise and Sport, 65, 377- 381.

Brewer, J., Balsom, P., Davis, J., \& Ekblom, B. (1992). The influence of birth date and physical development on the selection of a male junior international soccer squad. Journal of Sports Sciences, 10, 561-562.

Brewer, J., Balsom, P., \& Davis, J. (1995). Seasonal birth distribution amongst European soccer players. Sports Exercise and Injury, 1, $154-157$.

Breznitz, Z., \& Teltsch, T. (1989). The effect of school entrance age on academic achievement and social-emotional adjustment of children: Follow-up study of fourth graders. Psychology in the Schools, 26(1), 62-68.

Cameron, M. B., \& Wilson B. J. (1990): The effects of chronological age, gender, and delay of entry on academic achievement and retention: Implications for academic redshirting. Psychology in the Schools, 27(3), 260-263.

Cobley, S. (2005). Older, bigger, better! the nature of the relative age effect in physical education. SCAPPS, 10 .

Cobley, S., Abraham, C., \& Baker, J. (2008). Relative age effects on physical education attainment and school sport representation. Physical Education \& Sport Pedagogy, 13(3), $267-$ 276.

Crosser, S. L. (1991). Summer birth date children: Kindergarten entrance age and academic achievement. Journal of Educational Research, 84(3), 140-146.

Daniel, T. E., \& Janssen, C. T. L. (1987). More on the relative age effect. CAHPER journal/Journal de l' ACSEPL (Gloucester, Ont.) 53(2), Mar/Apr-mars/avr, 21-24.

Davis, B. D., Trimble, C. S., \& Vincent, D. R. (1980). Does age of entrance affect school achievement. The elementary School Journal, 80, 133-143.

Delorme, N., Boiché, J., \& Raspaud, M. (2010). Relative age effect in elite sports: Methodological bias or real discrimination? European Journal of Sport Science, 10, 91-8 96.

Delorme, N., Boich, J., \& Raspaud, M. (2010). Relative age effect in females sport: a diachronic examination of soccer players. Scandinavian Journal of Medicine \& Science in Sports, 20(3), 509515 .

DeMeis, J., \& Stearns, E. (1992). Relationship of school entrance age to academic and social performance. Journal of Educational Research, $86,21-27$.

Dhuey, E., \& Lipscomb, S. (2008): What makes a leader? Relative age and high school leadership. Economics of education Review, 27, 173-183.

Diamond, G. H. (1983). The birthdate effect. a madurational effect? Journal of learning disabilities, 16(3), 161-164.

Dietz, C., \& Wilson B. J. (1986): Beginning school age and achievement. Psychology in the Schools, 22(1), 93-94.

Dixon, J., Horton, S., \& Weir, P. (2011). Relative Age Effects: implications for leadership development. Intenational Journal of Sport \& Society, 2(2), 1-15.

Dudink, A. (1994). Birth date and sporting success. Nature, 368, 592.

Edgar, S., \& O’Donoghue, P. (2005). Season of birth distribution of elite tennis players. Journal of Sports Science, 23(10), 1013-1020.

Edwards, S. (1994). [Letter to the Editor]. Born too late to win? Nature, $370,186$.

Esteva, S., Drobnic, F., Puigdellivol, J., Serratosa, S., \& Chamorro, M (2006): Fecha de nacimiento y éxito en el baloncesto español. Apunts Medicina de l'Esport, 41,25-30.

Garcia Alvarez, V., \& Salvadores Canedo, J. (2005). El Efecto Relativo de la Edad en el Futbol. Training fútbol, 115, 36-42.

Garner, R. (1991). Children's use of strategies in reading. In D. F. Bjorklund (Ed.), Children's strategies: Contemporary views of cognitive development (pp. 245-268). Hillsdale, NJ: Erlbaum.
Giacomini, C. P. (1999). Association of birthdate with success of nationally ranked junior tennis players in the United States. Perceptual and motor skills, 89(2), 381-386.

Gilly, M. (1965): Moisse de naissance et réussite scolaire. Enfance, 4, 491-503.

Glamser, F. D., \& Vincent, J. (2004). The relative age effect among elite American youth soccer players. Journal of sport behaviour, 27(1), 31-38.

Glamser, F. D., \& Marciani, L. M. (1992). The birthdate effect and college athletic participation: some comparisons. Journal of sport behavior, 15(3), Sept, 227-238.

Gómez Piqueras, P. (2009). El efecto de la edad relativa en las categorías inferiores de los equipos de fútbol y su continuidad en el alto nivel. Abfútbol: revista técnica especializada en fútbol, 40, 79-87.

Gonzalez Aramendi, J. M. (2004). Mes de nacimiento y éxito en el fútbol. Estudio del Efecto Relativo de la Edad en el fútbol guipuzcoano. Euskonews \& Media.

González Aramendi, J. M. (2007). El efecto relativo de la edad en el fútbol. Archivos de Medicina del Deporte, 24(117), 5-13.

Grondin, S., Deshaies, P., \& Nault, L. P. (1984). Trimestres de naissance et participation au hockey et au volleyball. La Revue Que becoise de l'Activite' Physique, 2, 97-103.

Grondin, S., Proulx, J., \& Zhou, Y. (1993). Fecha de naissance et scolaire del rendement. Apprentissage et socialización, 16, 169-174.

Grondin, S., \& Koren, S. (2000). The relative age effect in professional baseball: A look at the history of Major League Baseball and at current status in Japan. Avante, 6, 64-74.

Grondin, S., \& Trudeau, F. (1991). Date de naissance et ligue nationale de hockey: Analyses en fonction de diffe'rents parame tres. Revue des Sciences et Techniques des Activite's Physiques et Sportives, 26, $37-$ 45 .

Gutiérrez Díaz del Campo, D. (2006). El efecto de la edad relativa en la formación del talento deportivo. Ponencia inédita en el Máster Universitario en Detección y Formación del Talento en Jóvenes Futbolistas organizado por la Federación Española de Fútbol y la universidad de Castilla-la Mancha. Madrid.

Gutiérrez Díaz del Campo, D. (2011). El efecto de la edad relativa en el deporte. Ponencia inédita en el Cuso de Experto en Director Deportivo organizado por la Liga Profesional de Fútbol y las universidades de Castilla-La Mancha y Oviedo. Madrid.

Gutierrez Díaz del Campo, D., Pastor Vicedo, J. C., González Víllora, S., \& Contreras Jordán, O. R. (2010). Relative age effect in youth soccer players from Spain. Journal of Sports Science and Medicine 9, 190198.«http://www.jssm.org/vol9/n2/5/v9n2-5text.php»

Hancock, D. J., Ste-Marie, D. M., \& Young, B. W. (en prensa). Coach selections and the relative age effect in male youth ice hockey. Research Quarterly for Exercise and Sport.

Hauk, A., \& Finch, A. (1993). The effect of relative age on achievement in middle school. Psychology in the Schools, 30, 74-79.

Helsen, W., Winckel, J. V., \& Willians, A. M. (2005). The relative age effect in youth soccer across Europe. Journal of sport Sciences, 23(6), 629-636.

Helsen, W. F., Janet L. Starkes, J. L., \& Winckel, J. V. (1998). The influence of relative age on success and dropout in male soccer players. American journal of human biology, 12, 729-735.

Helsen, W. F., Janet, L., Starkes, J. L., \& Winckel, J. V. (2000). Effect of a change in selection year on success in male soccer players. American journal of human biology, 12, 729-735.

Horn, R. R., \& Okumura, M. (2011). It's time to eliminate the relative age effect in American soccer. Soccer Journal, 56(2), 38-40.

Hurley, W., Lior, D., \& Tracze, S. (2001). A Proposal to Reduce the Age Discrimination in Canadian Minor Hockey. Canadian Public Policy, 27(1), 65.

Pérez Jiménez, I., \& Matthew, G. P. (2008). Relative age effect in Spanish association football: Its extent and implications for wasted potential. Journal of Sports Sciences 26, 995-1003.

Kaiserman, K. (2005). Skill Based Division of Talent in Recreational Youth Leagues. www.sportskids.com/nl/newsletter/ newsletter1_archive.asp.

Kinard E. M., \& Reinhertz, H. (1986). Birthdate effects on school performance and adjustment: A longitudinal study. Journal of Educational Research, 79(6), 366-372.

Krouse, W. (1995). The relative age effect and elite status in the national hockey league. Unpublished manuscript. Ontario, Canada: Wilfrid Laurier University. 
Landers, M., \& Fine, G. (1996). Learning life's lessons in tee ball: The reinforcement of gender and status in kindergarten sport. Sociology of Sport Journal, 13, 87-93.

Lesma, M. L., Pérez González, B., \& Salinero, J. J. (2011). El efecto de la edad relativa (RAE) en la liga de fútbl española. Journal of Sport and Health Research, 3(1), 35-46.

Lien, L., Tambs, K., Oppedal, B., Heyerdahl, S., \& Bjertness, S. (2005). Is relatively young age within a school year a risk factor for mental health problems and poor school performance? A population based cross sectional study of adolescents in Oslo, Norway. www.Biomedcentral.com.

Livingston, R. Balkozar, S. A., \& Bracha, S. (1993). Season of birth and neurodevelopmental disorders: summer birth is associated with dyslexia. Journal Of The American Academy Of Child And Adolescent Psychiatry, 32, 612-616.

Maddux, C. D. (1980). First grade entry age in a sample of children labeled learning disabled. Learning Disability Quarterly, 3, 79-83.

Maddux, C. D., Stacy, D., \& Scott, M. (1981). School entry age in a group of gifted children. Gifted Child Quartery, 25, 180-184.

Malina, R. (1994). Physical growth and biological maturation of young athletes. Exercise and Sport Sciences Reviews, 22, 389-434.

Malina, R. (2003). Growth and maturation of young football players: implications for selection, performance and competitions. World congress on science and football. Lisbon 11-15 April.

Malina, R., Bouchard, C., \& Bar-Or, O. (2004). Growth, maturation and physical activity. Human kinetics.

Martín Acero, R., Fernández del Olmo, M., Lago Peñas, C., Vizcaya Pérez, F., Rodríguez Gómez, A., \& Macario Bravo, F. (2007) El efecto relativo de la edad en la Metodología del Rendimiento con jóvenes futbolistas. RED, Revista de Entrenamiento Deportivo, Tomo XIII, n'3.

Martín Acero, R., \& Lago Peñas, C. (2005). Deportes de Equipo: Comprender la Compejidad para aumentar el rendimiento. INDE. Barcelona.

Martin, R. P., Foels, P., Clanton, G., \& Moon, K. (2004): Season of birth is related to child retention rates, achievement, and rate of diagnosis of specific LD. Journal of Learning Disabilities, 37(4), 307-17.

Medic, N., Young, B. W., Starkes, J. L., Weir, P. L., \& Grove, J. (2009). Gender, age, and sport differences in relative age effects among US Masters swimming and track and field athletes. Journal of Sports Sciences, 27(14), 1535-1544.

Miller, P. H. (1991). The development of strategies in selective attention In D. F. Bjorklund (Ed.), Children's strategies: Contemporary views of cognitive development (pp. 157-184). Hillsdale, NJ: Erlbaum.

Musch, J., \& Grondin, S. (2001): Unequal competition as an impediment to personal development: a review of the relative age effect in sport. Developmental review, 21(2), June, 147-167.

Musch, J., \& Hay, R. (1999). The relative age effect in soccer: cross cultural evidence for a systematic discrimination against children born late in the competition year. Sociology of Sport Journal, 16, 5464

Nakata, H., \& Sakamoto, K. (2011). Relative age effect in Japanese male athletes. Perceptual \& Motor Skills, 113(2), 570-574.

Peck, B., \& Trimmer, K. (1995). The late birthday effect in Western Australia. Issues in Educational Research, 5(1), 35-52.

Pellegrini, A. (1992). Kindergarten children's social-cognitive status as a predictor of first-grade success. Early Childhood Research Quarterly, 7, 565-577.

Portella, D. L., de Arruda, M., \& Cossio-Bolaños M. A. (2011). Valoración del rendimiento físico de jóvenes futbolistas en función de la edad cronológica. Apunts: Educación física y deportes, 106, 42-49.

Reilly, J., Bangsbo, J., \& Franks, A. (2000). Anthropometric and physiological predispositions for elite soccer. Journal of Sports Sciences, 18, 669-683

Rejeski, W., Darracott, C., \& Hutslar, S. (1979). Pygmalion in youth sport: A field study. Journal of Sport Psychology, 1, 311-319.

Roberts, S. J., \& Fairclough, S. J. (2012). The Influence of Relative Age Effect in the Assessment of High School Students in Physical Education in the United Kingdom. Journal Of Teaching In Physical Education, 31(1), 56-70

Roberts, S. J., Boddy, L. M., Fairclough, S. J., \& Stratton, G. (2012). The Influence of Relative Age Effects on the Cardiorespiratory Fitness Levels of Children Age 9 to 10 and 11 to 12 Years of Age. Pediatric Exercise Science, 24(1), 72-83.
Ryan, P. (1989). The relative age effect on minor sport participation. Unpublished master's thesis. Montreal, Quebec: McGill University.

Schorer, J., Neumann, J., Cobley, S. P., Tietjens, M., \& Baker, J. (2011). Lingering Effects of Relative Age in Basketball Players' Post Athletic Career. International Journal Of Sports Science \& Coaching, 6(1), 143-148.

Siegler, R. S. (1991). Children's thinking. Englewood Cliffs, NJ: Prentice Hall.

Simmons,C., \& Paull, G.C. (2001). Season of birth bias in association football. Journal of Sports Sciences, 9(9), 677-686.

Spitzer, S., Cupp, R., \& Parke, R. D. (1995). School entrance age, social acceptance, and self-perception in kindergarten and 1st grade. Early Childhood Research Quarterly, 10(4), 433-450.

Sprietsma, M. (2006). The effect of relative age in the first grade of primary school on long-term scholastic results international. Comparative evidence using PISA 2003. International conference «economics of education: major contributions and future directions». Dijon.

Stanaway, K. B., \& Hines, T. M. (1995). Lack of Season of Birth Effect Among American Athletes. Perceptual and motor skills, 81(1), 952954.

Strøm, B. (2004) Student achievement and birthday effects. Mimeo, Norwegian University of Science and Technology (online), (21 screens/inclusive page). Descargado desde: http://www.hks.harvard.edu.

Thompson, A. H., Barnsley, R. H., \& Stebelsky, G. (1991): «Born to play ball» The relative age effect and Major League baseball. Sociology of Sport Journal, 8(2), 146-151.

Thompson, A., Barnsley, R., \& Stebelsky, G. (1992). Baseball performance and the relative age effect: Does Little League neutralize birthdate selection bias? Nine, 1, 19-30.

Thompson, A. H., Barnsley, R. H., \& Dyck, R. J. (1999). A new factor in youth suicide: the relative age effect. Canadian Journal of Psychiatry, 44(1), 82-85.

Thompson, A. H., Barnsley, R., \& Battle, J. (2004). The relative age effect and the development of self-esteem. Educational Research, 46(3), 313-320.

Thompson, D. (1971). Season of birth and success in the secondary school. Educational Research, 14, 56-60.

Vaeyens, R., Philippaerts, R. M., \& Malina, R. M. (2005). The relative age effect in soccer: A match-related perspective. Journal Of Sports Sciences, 23(7), 747-756.

Verhulst, J. (1992). Seasonal birth distribution of West European soccer players: A possible explanation. Medical Hypotheses, 38, 346-348.

Ward, P., \& Williams, A. M. (2005). Perceptual and Cognitive Skill Development in Soccer: The Multidimensional Nature of Expert Performance. Journal of Sport \& Exercise Psychology, 25(1), 93111.

Wilson, G. (1999): The birthdate effect in school sports teams. European journal of physical education (Reading, England) 4(2), 139-145.

${ }^{2}$ Ejemplos de proyectos los encontramos en Gran Bretaña, en la universidad de Loughborough http://www.lboro.ac.uk/departments/ssehs/research/sport-science/youth-sport/research/researcharchive-related/relative-age-effect.html y en South West Talent Development Centre http://www.cornwallsportspartnership.co.uk/files/relative age effect.pdf

${ }^{3}$ http:/www rfefes/FCKeditor/UserFiles/File/dipticos/dipticomatententos pdf

${ }^{4} \mathrm{http} \cdot / /$ directo uniovi.es/postorado/cabecera e . asp? Curso $=2011 \&$ IdPrograma $=7359$

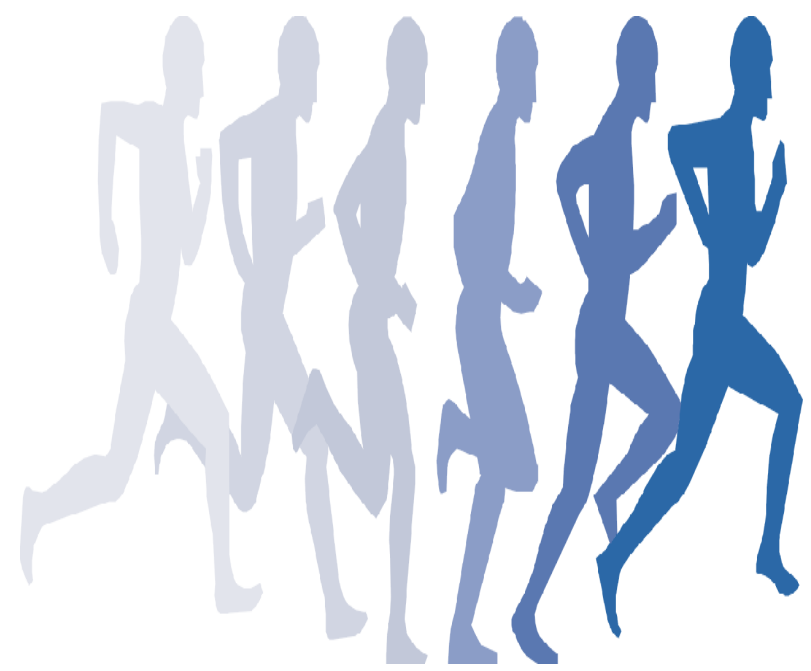

